REVISTA DE DERECHO UNED, NÚM. 28, 2021

\title{
LA PARTICIPACIÓN DE TERCEROS EN EL ARBITRAJE INTERNACIONAL
}

\section{THE PARTICIPATION OF THIRD PARTIES IN INTERNATIONAL ARBITRATION}

Nayiber Febles Pozo ${ }^{1}$

\begin{abstract}
Sumario: I. Introducción. II. Consideraciones en torno a la figura procesal del amicus curiae y el tercero financiador. III. La financiación por terceros en el arbitraje internacional. III.1. El acuerdo de financiación con terceros y su influencia en el procedimiento arbitral ¿Está sujeto el acuerdo de financiación a normas de transparencia y a obligación de divulgación? III.1.A. La transparencia en el arbitraje y el acuerdo de financiación con terceros: el deber de revelación del acuerdo de financiación.. III.1.B. El deber de divulgación del acuerdo de financiación en las reglas arbitrales y tratados internacionales. Consideraciones desde el ámbito comparado. IV. Los efectos de la financiación por terceros en el arbitraje internacional. V. CONCLUSIONES. VI. Bibliografía.
\end{abstract}

Resumen: La transparencia en el arbitraje internacional, comercial y de inversiones, es un tema relevante y de actualidad. En el presente trabajo realizamos un análisis de la participación de terceros desde la perspectiva de la transparencia en el procedimiento arbitral. Para ello tendremos en cuenta la figura procesal del amicus curiae y la financiación por terceros. Respecto al tercero financiador en el arbitraje internacional, existe un conglomerado de cuestiones de vital importancia en la actualidad, entre los que podemos citar: la divulgación del acuerdo de financiación; los efectos de la

1 Profesor Contratado Doctor (Acred.) de Derecho internacional privado en la Universidad Nacional de Educación a Distancia. UNED (nfeblespozo@der.uned.es).

(C) UNED. Revista de Derecho UNED, núm. 28, 2021 
financiación por terceros, los cuales serán objetos de análisis en el presente trabajo.

Palabras claves: Participación de terceros, amicus curiae, arbitraje internacional, financiación por terceros.

ABSTRACT: Transparency in international, commercial and investment arbitration is an issue of the greatest relevance and relevance. In this work we carry out an analysis of the participation of third parties, from the perspective of transparency in the arbitration procedure. For this we will take into account the procedural figure of the amicus curiae and the financing by third parties. Regarding the third party financier in international arbitration, there is a conglomerate of issues of vital importance today, among which we can mention: the disclosure of the financing agreement; the effects of financing by third parties, which will be analyzed in this work from a practical perspective through case analysis.

Keywords: Third party participation, amicus curiae, international arbitration, third party financing.

Recepción original: 07-04-2021

Aceptación original:27-09-2021

\section{INTRODUCCIÓN}

En el ámbito del Derecho público y privado, el carácter público de los procedimientos judiciales y el principio de imparcialidad judicial son inherentes al Estado de Derecho, en el cual el acceso y el conocimiento público de los procedimientos judiciales proporciona un medio para garantizar la confianza pública en el sistema jurídico. Sin embargo, dichos principios no son aplicados en el arbitraje. En el abritraje, por contra, el punto de partida para el acceso a las audiencias es completamente ajeno al Derecho público. Los procedimientos arbitrales son privados, no hay derecho de acceso a nadie más que a las partes, incluso en la actualidad la noción implícita de confidencialidad en el arbitraje internacional ya no puede darse por sentado, siendo muy discutible en la práctica la privacidad de las audiencias ${ }^{2}$.

2 CARON, D., CAPLAN, L.M., The UNCITRAL Arbitration Rules: A Commentary, Segunda Edición, Oxford, 2013, p. 607. 
En diferentes medidas los tribunales arbitrales han ido ganando facultades discrecionales ${ }^{3}$ en relación con la participación de terceros en el procedimiento arbitral, especialmente en el arbitraje inversor-Estado (ISDS) ${ }^{4}$. Tanto es así, que la participación de terceros es un tema que hoy se discute bajo la rúbrica de transparencia. El presente trabajo tiene por objeto analizar la participación de terceros en el arbitraje internacional, teniendo en cuenta las reglas arbitrales a nivel internacional como son: las reglas del Centro Internacional de Arreglos de Diferencias Relativas a Inversiones (CIADI), las reglas de la Comisión de las Naciones Unidas para el Derecho Mercantil Internacional (CNUDMI) y los Tratados Bilaterales de Inversión (TBI) y en el propio procedimiento arbitral.

\section{CONSIDERACIONES EN TORNO A LA FIGURA PROCESAL DEL AMICUS CURIAE Y EL TERCERO FINANCIADOR}

En el contexto del Tratado de Libre Comercio de América del Norte (TLCAN) son admisibles las comunicaciones de "no partes o no Contendientes" como las presentaciones de amicus curiae y las comunicaciones del Artículo 1128 por los Estados parte no demandantes, convirtiéndolo en referente internacional. La cuestión de si el amicus curiae está disponible en los arbitrajes del Capítulo Once del TLCAN se planteó por primera vez en el caso Methanex Corp. vs. United States ${ }^{5}$. En el cual, se sostuvo que el artículo 15.1 del Reglamento de la CNUDMI (actualmente artículo 17.1 del Reglamento de la CNUDMI modificado en el 2010), incluía el derecho a presentar una solicitud de amicus curiae en el ISDS. El actual artículo 17.1 es una de las disposiciones fundamentales del Reglamento de la CNUDMI y establece esencialmente que todas las partes deben ser tratadas con igualdad y que las mismas, en cualquier etapa del procedimiento, pueden hacer valer sus derechos ${ }^{6}$. El derecho a presentar memorias

3 Cuestión que es reafirmada por PASCUAL VIVES, refiriéndose a uno de sus trabajos en, PASCUAL VIVES F.J., "La institución del amicus curiae y el arbitraje de inversiones", Anuario español de derecho internacional privado, Vol. 27, 2011, p. 352.

${ }_{4}$ Puede verse una lista detalla de casos en SUBRAMANIAN, S.R., "The Participation of Amicus Curiae in Investment Treaty Arbitration", Journal of Civil \& Legal Sciences, Vol. 5, No. 4, 2016, p. 205.

5 Methanex Corp. vs. United States of America, In the Matter of an Arbitration Under Chapter 11 of the North American Free Trade Agreement and the UNCITRAL Arbitration Rules, 1999. Disponible en: https://www.italaw.com/cases/683, consultado el 5/07/2020.

$6 \quad$ El actual Art. 17.1 del Reglamento de la CNUDMI establece: "Con sujeción a lo dispuesto en el presente Reglamento, el tribunal arbitral podrá dirigir el arbitraje del modo que considere apropiado, siempre que se trate a las partes con igualdad y que en

(C) UNED. Revista de Derecho UNED, núm. 28, 2021 
de amicus curiae no está expresamente establecido en el artículo y, por lo tanto, el tribunal del caso Methanex Corp. vs. United States, en relación con el extinto artículo 15.1, escuchó argumentos vigorosos sobre si el amicus curiae fue autorizado por esa disposición ${ }^{7}$.

Mientras que Canadá y Estados Unidos argumentaron que el amicus curiae estaba comprendido en la extinta Regla 15, México y el reclamante, Methanex, argumentaron que el derecho a presentar un amicus curiae era un derecho sustantivo que no podía leerse en una disposición procesal como el artículo 15.1. En conclusión: el tribunal sostuvo que el artículo 15.1 permitía la participación de amicus curiae. Decisión que fue posteriormente reafirmada por el tribunal del caso UPS vs. Canadá ${ }^{8}$.

La participación de amicus curiae plantea preocupaciones algo más complejas que las audiencias abiertas y la publicación de laudos, debido a la influencia que pueden tener respecto del alcance, la complejidad y la duración de un procedimiento arbitral. En este sentido, el éxito de los amicus curiae a nivel internacional depende del tipo de tribunal en el cual han intentado participar, ya que en algunos la participación del amicus curiae ha sido severamente limitada9

Durante las últimas décadas, el acceso público a las audiencias, la documentación, así como la participación de amicus curiae durante el proceso arbitral, como forma de incentivar la transparencia, ha tenido su respaldo a través de normativas que rigen el arbitraje en el contexto internacional, dentro de las cuales podemos citar las enmiendas de 2006 al Reglamento de Arbitraje del CIADI, las Reglas de Transparencia de la CNUDMI, la Convención de Mauricio sobre la transparencia y la práctica reciente de un gran número de tratados firmados por un amplio número de Estados $^{10}$. Pero queda un

una etapa apropiada del procedimiento se dé a cada una de las partes una oportunidad razonable de hacer valer sus derechos. En el ejercicio de su discrecionalidad, el tribunal arbitral dirigirá las actuaciones con miras a evitar demoras y gastos innecesarios y a llegar a una solución justa y eficaz del litigio entre las partes".

Methanex Corp. vs. United States, Decision of the Tribunal on Petitions from Third Persons to Intervene as Amici Curiae, 2001, paragraph 31.

8 UPS vs. Canada, Decision of the Tribunal on Petitions for Intervention and Participation as Amici Curiae, 2001, paragraph 39; Véase también, Glamis Gold Ltd vs. USA, Arbitration under Chapter 11 of the NAFTA, UNCITRAl Arbitration Rule, 2005.

9 BASTIN, L., "Amici Curiae in Investor-State Arbitration: Eight Recent Trends", Arbitration International, Vol. 30, No. 1, 2014, p. 125.

10 Aunque mucho se ha discutido sobre el desarrollo de la transparencia en el marco del TLCAN, el CIADI y la CNUDMI, al respecto véase JAMES, D.F., ODYSSEUS, G.R., "Towards a New World for Investor-State Arbitration through Transparency", N.Y.U. J.Int'l.L, Vol. 48, 2015, pp., 811-818; JANSEN CALAMITA, N., 
largo camino por recorrer. En el ISDS, la participación de amicus curiae ha sido significativa, teniendo cada vez mayor relevancia y presencia en el procedimiento, tal como lo demuestran varios casos de arbitraje de inversiones ${ }^{11}$. Sin embargo, siguen existiendo algunos vestigios de disconformidad y reticencia ${ }^{12}$ a la institución del amicus curiae, el cual es visto como una carga adicional para las partes en controversia. Su participación conlleva a la presentación de una nueva serie de alegatos, lo que puede provocar que la participación de los amicus curiae no siempre sea tenida en cuenta y que sus presentaciones puedan resultar determinantes en el sentido del laudo.

Es el caso del laudo final del mencionado caso Methanex Corp. vs. United States donde el tribunal señaló que el amicus curiae era útil, pero el laudo no dejó claro si se tuvo en cuenta la participación del amicus curiae ${ }^{13}$ y, de ser así, cómo se llevó a cabo. En la práctica actual, se puede apreciar que, aunque algunos laudos reconocen la presencia del amicus curiae a través de una mención expresa del mismo o de un resumen del contenido del escrito presentado, los tribunales arbitrales han sido muy cautelosos o selectivos para expresar en su propio razonamiento sustantivo la influencia que ha tenido el escrito o comunicación del amicus curiae ${ }^{14}$.

"Dispute Settlement Transparency in Europe's Evolving Investment Treaty Policy”, J.World Inv. \& Trade, Vol. 15, 2014, pp. 653-655; CHRISTINA, K., AUGUST, R., "Transparency versus Confidentiality in International Investment Arbitration The Biwater Gauff Compromise", Law \& Prac. Int'L cts and Tribunals, Vol. 6, 2007, pp. 101-103. En este sentido, debemos tener presente también la adopción de los Principios de Inversión Gubernamental del G20, Objetivo de la política de "transparencia" en la solución de diferencias internacionales de inversión.

${ }_{11} \mathrm{Al}$ respecto, véase el resumen de las decisiones de tribunales inversor-Estado hasta el año 2012 en, BASTIN, L., "The Amicus Curiae in Investor-State Arbitration", Camb.J. Int'l \& Comp.L, Vol. 1, 2012, pp. 214-223.

12 SOMMER, CH, G., "Los alcances del amicus curiae en el arbitraje internacional de inversiones", Revista de la Facultad Universidad Nacional de Córdoba, Vol. 2, No. 2, 2011, p. 172.

13 Véase, Methanex Corp. vs. United States, Final Award, 2005, paragraph 11, "From this Award and from the Partial Award also, it will be evident that the Tribunal has relied heavily on the submissions of Counsel, who were assisted by many others whose names do not appear on the transcript of the hearings. In adversarial proceedings addressing such a massive, complicated and difficult dispute over many years, it could not be otherwise. At the beginning of this Award, therefore, it is appropriate to record our appreciation of the scholarship and industry which Counsel for the Disputing Parties, Mexico and Canada as NAFTA Parties and the amici have deployed during these lengthy arbitration proceedings, together with their respective experts, assistants and other advisers". Consúltese también, SARAVANAN, A., SUBRAMANIAN, S.R., "The Participation of Amicus Curiae in Investment Treaty Arbitration", Journal of Civil \& Legal Sciences, Vol. 5, No. 4, 2016, pp. 202-203.

14 LAMB, S., HARRISON, D., HEW, J., "Recent developments in the law and practice of amicus briefs in investorstate arbitration", Indian Journal of Arbitration 
Las críticas y preocupaciones en relación con la participación del amicus curiae en el contexto ISDS han sido exclusivamente pro-gubernamentales y anti-inversionistas. Críticas que consideramos infundadas, ya que la participación del amicus curiae puede aportar valiosos elementos al procedimiento arbitral que, además, no siempre tienen vinculación con los gobiernos ni con los intereses del inversor. Los amicus curiae no son solamente ONGs, las cuales han sido consideradas como "el paradigma clásico de participación de amicus curiae en defensa del derecho de regulación de los Estados contra las medidas regulatorias impugnadas en el arbitraje"15. El reconocimiento del derecho de participación del amicus curiae debe tenerse en cuenta desde una variedad de elementos presentes en el litigio, como pueden ser la complejidad del caso, su repercusión en la sociedad, su relación o incompatibilidad con algún derecho fundamental regulado en el Estado receptor de la inversión, entre otros.

Debe tenerse en cuenta que los amicus curiae no siempre solicitan participar en un procedimiento para aportar elementos en defensa del demandado, existen casos en los que los amicus curiae han presentado argumentos relevantes en apoyo al demandante. Por ejemplo, Grand River Enterprises Six Nations Ltd and Others vs. United States of America ${ }^{16}$, Apotex Inc. vs. United States of America ${ }^{17}$ y Apotex Holdings Inc., Apotex Inc. vs. United States of America ${ }^{18}$. Incluso, en ocasiones, asumen una posición neutral, o sea, para esclarecer, dilucidar, una cuestión jurídica para el tribunal y no precisamente para apoyar a las partes en litigio, tal y como sucedió en los

Law, Vol. 5, No. 2, 2017, p. 85; SHIKAWA T., "Third Party Participation in Investment Treaty Arbitration", International and Comparative Law Quarterly, Vol. 59, No 2, 2010, pp, 409-411; DE BRABANDERE, E., "Human Rights Considerations in International Investment Arbitration", en FITZMAURICE, M., MERKOURIS, P. (Eds), The Interpretation and Application of the European Convention of Human Rights: Legal and Practical Implications, Queen Mary Studies in International Law, London, 2012, pp. 190-215.

${ }_{15}$ BASTIN, L., Amici Curiae in Investor-State Arbitration: Eight Recent Trends...op.cit...nota 8, p. 135.

16 Grand River Enterprises Six Nations Ltd and Others vs. United States of America, UNCITRAL (NAFTA), 2009. Amicus Curiae Submission of the Office of the National Chief of the Assembly of First Nations. Disponible en: https://www.italaw. com/cases/510, consultado el 15/07/2020.

17 Apotex Inc. vs. United States of America, UNCITRAL (NAFTA), Procedural Order No. 2, Participation of a Non-Disputing Party, 2011, paragraph 10.

18 Apotex Holdings Inc., Apotex Inc. vs. United States of America, ICSID Case No. ARB(AF)/12/1, Procedural Order on the Participation of the Applicant, BNM, as a Non-Disputing Party, paragraph 25, 2013. Disponible en: https://www.italaw.com/ cases/1687, consultado el 15/07/2020. 
casos, Electrabel SA vs. Republic of Hungary ${ }^{19}$, AES Summit Generation Limited \& Another vs. Republic of Hungary ${ }^{20}$ y Eureko vs. Slovak Republic ${ }^{21}$.

El derecho de participación del amicus curiae en el procedimiento arbitral no resulta tarea fácil. Cuando un amicus curiae muestra cierto interés en un caso determinado y solicita participar en el procedimiento, el camino se le hace muy escabroso y complicado, son más los obstáculos e impedimentos a los que deben enfrentarse que las posibilidades reales de participación. Tanto es así, que ante una solicitud de participación de un amicus curiae, el tribunal, por lo general, debe tener en cuenta los siguientes aspectos: el tratado en virtud del cual se llevó a cabo la inversión o se presenta el procedimiento arbitral; las reglas que rigen el procedimiento; el ámbito específico de participación y los posibles beneficios que aportaría al tribunal, los cuales deben ser distintos a los aportados por las partes. Sin duda alguna, se dificulta encarecidamente la participación del amicus curiae en el procedimiento arbitral. Es por ello, que resulta necesario, tanto a nivel institucional como internacionalmente, crear mecanismos más sencillos y eficaces que, desde una perspectiva amplia, global, permitan una mayor participación de los amicus curiae en el procedimiento.

A pesar de la importancia que ha adquirido la figura procesal del amicus curiae a nivel internacional, España carece de una regulación específica de dicha institución que legitime la participación

19 Electrabel SA vs. Republic of Hungary, ICSID Case No. ARB/07/19, Laudo 2015, paragraph 234, "Second, the Commission's submissions to the Tribunal (as a "non-disputing party") raised important and extensive issues of jurisdiction and applicable law. These were not issues, particularly as to jurisdiction, raised by Hungary. However, Electrabel had to expend much time and cost to deal with the Commission's submissions. In effect, far from exercising the traditional role of an "amicus curiae", the Commission became a second respondent more hostile to Electrabel than Hungary itself. If accepted by the Tribunal, the Commission's submissions would have been fatal to Electrabel's case. The Tribunal was required to decide these issues at length in its Decision and there reject a material part of the Commission's submissions. Overall, the Commission's participation in this arbitration was a hugely complicating factor, as were to a lesser extent the pending legal proceedings in Luxembourg (being only concluded on 1 October 2015). For all these, Electrabel bore by far the greatest burden". Disponible en: https://www.italaw.com/cases/380, consultado el 15/07/2020.

20 AES Summit Generation Limited \& Another vs. Republic of Hungary, ICSID Case No. ARB/07/22, 2010. Disponible en: https://www.italaw.com/cases/193, consultado el 15/07/2020.

${ }_{21}$ Eureko vs. Slovak Republic, PCA Case No. 2008-13, Award on Jurisdiction, Arbitrability and Suspension, Section V, 2010. Disponible en: https://www.italaw. com/cases/documents/418, consultado el 15/07/2020. 
de terceros en el procedimiento ${ }^{22}$. Con todo ello, alguna institución de arbitraje española, si bien no ha regulado específicamente la figura del amicus curiae, ha establecido en su respectivo reglamento la participación de terceros en el procedimiento. Por ejemplo, el Reglamento de la Corte de Arbitraje de Madrid (CAM) establece: "Los árbitros podrán, a petición de cualquiera de las partes oídas todas ellas. Admitir la intervención de uno o más terceros como partes en el

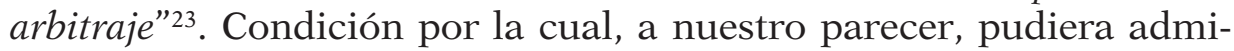
tirse la participación del amicus curiae en el arbitraje. Sin embargo, debe reconocerse que la figura del amicus curiae ha tenido más prevalencia en el arbitraje de inversiones, procedimiento del que comúnmente suele conocer el CIADI como institución, careciendo España de institución arbitral con tradición en la administración de dichos procedimientos.

No obstante, la participación de la figura procesal del amicus curiae en el arbitraje de inversiones se justifica por la presencia del interés público ${ }^{24}$ presente en este tipo de arbitraje, que siempre buscan promover el interés colectivo presente por encima del interés privado del inversor. La presencia del amicus curiae en el procedimiento puede contribuir a la existencia de más transparencia en el mismo, siempre que incida en la búsqueda de una mayor apertura procesal, divulgación pública de la información y menos secretismo del procedimiento ${ }^{25}$. Esto puede favorecer, a su vez, una mayor legitimidad del sistema ${ }^{26}$, teniendo en cuenta que la participación pública está severamente limitada en el arbitraje de inversiones. Además, por los efectos que tiene la resolución final del litigio no solo en el erario de los Estados, sino también directamente en la población ${ }^{27}$.

22 BAUER BRONSTRUP, F., "El amicus curiae en la jurisdicción constitucional española”, Revista Española de Derecho Constitucional, No. 108, 2016, pp. 185-188.

23 Art. 9.2 del Reglamento de Arbitraje de la Corte de Arbitraje de Madrid.

24 FEBLES POZO, N, "La transparencia y el interés público en el arbitraje de inversiones", Vniversitas, Vol. 69, 2020, p. 4.

${ }_{25}$ LEVINE, E., "Amicus Curiae in International Investment Arbitration: The Implications of an Increase in Third-Party Participation", Berkeley Journal of International Law, No. 29, 2011, p. 217.

26 MARSHALL, F., MANN, H., "Good Governance and the Rule of Law: Express Rules for investor-state Arbitrations Required”, International Institute for Sustainable Development Submissions, 2006, p. 3.

27 FACH GÓMEZ, K., "Rethinking the role of amicus curiae in international investment arbitration: how to draw the line favorably for the public interest", Fordham International Law Journal, Vol. 35, 2012, p. 524. 
Con todo, creemos que la simple participación de un tercero en el procedimiento arbitral no significa que el procedimiento resulte necesariamente más transparente. Por nuestra parte, consideramos que la participación de terceros puede ser un factor importante, un mecanismo procedimental en la búsqueda de una mayor transparencia del proceso, un indicador del nivel de transparencia dentro del ISDS; pero no el elemento esencial. El núcleo de la transparencia en el procedimiento es la divulgación, el acceso y conocimiento público de todas las actuaciones que se llevan a cabo durante el procedimiento. De ahí que la doctrina reconozca que, en cuanto a la transparencia en el ISDS, existen al menos tres aspectos procesales básicos que pueden verse afectados cuando la controversia se resuelve mediante arbitraje internacional ${ }^{28}$. Estos se refieren al conocimiento de la disputa, el acceso al procedimiento y la información relativa a su resultado final ${ }^{29}$. Pero el arbitraje de inversiones no se ha emancipado del todo del carácter confidencial y secreto del proceso de arbitraje comercial internacional. No todos los escritos y las audiencias orales suelen estar disponibles o accesibles al público, y las decisiones finales del tribunal solo se publican con el consentimiento de las partes. Como resultado, el público, en la inmensa mayoría de los casos, desconoce todo cuanto acontece en el procedimiento, incluyendo la resolución de estos.

La participación pública en el proceso de arbitraje de inversiones es más a menudo la excepción que la norma. Tanto es así, que algunos autores consideran que el arbitraje de inversiones es una forma de control de las funciones gubernativas de los propios Estados. Para algunos autores, el arbitraje de inversiones es considerado como una especie de "ley administrativa global" 30 , lo que hace necesario una actualización del sistema y una mayor participación de terceros en el procedimiento.

La participación de terceros no solo puede propiciar la búsqueda de una mayor transparencia en el procedimiento arbitral, sino también, de forma general, contribuir a mejorar la sistemática del Derecho internacional de las inversiones y una mejor evolución

28 POLANCO LAZO, R., "El capítulo de inversiones en el acuerdo de asociación transpacífico. ¿Una posibilidad de cambio y convergencia?”, 2016, p. 14. Disponible en: http://ssrn.com/abstract=2515216, consultado el 15/07/2020.

29 ORTINO, F., "External Transparency of Investment Awards", Inaugural Conference of the Society for International Economic Law (SIEL): New Horizons of International Economic Law, Geneva, 2008. Dispoible en: http://www.ssrn.com/link/SIELInaugural-Conference.html, consultado el 15/07/2020.

30 VAN HARTEN, G., LOUGHLIN, M.: "Investment Treaty Arbitration as a Species of Global Administrative Law”, Eur.J.Int'l. L., Vol. 17, No. 1, 2006, p. 121.

(C) UNED. Revista de Derecho UNED, núm. 28, 2021 
de este. En este sentido, se permitirá lograr una coherencia entre el Derecho internacional de inversión y el Derecho internacional en general, teniendo en cuenta la trascendencia de las cuestiones que son tratadas bajo el paraguas protector del primero en regímenes de tratados especiales y con una innegable influencia en el Derecho internacional de los Derechos Humanos, o en el Derecho ambiental, por solo citar algunos. En relación con esto último, PETERSMANN habla de "la fragmentación y los conflictos entre diferentes regímenes de tratados internacionales especiales" ${ }^{31}$. Todo ello puede llevar a que los propios Estados, los gobiernos nacionales, se encuentren cada vez más sujetos al control de instituciones internacionales o supranacionales.

El sistema de participación de terceros en el procedimiento arbitral se encuentra, en la actualidad, fragmentado, carente de un enfoque sistemático que permita un mejor tratamiento en cuanto a la participación del amicus curiae en el arbitraje de inversiones. Ante la evidencia de algunas iniciativas desarrolladas al efecto, como son las modificaciones de las reglas del CIADI, el TLCAN, el modelo canadiense de TBI, el Reglamento sobre la Transparencia de la CNUDMI, que se limitan exclusivamente a la presentación de comunicaciones o informes que no son jurídicamente vinculantes. Sin embargo, el modelo canadiense de TBI y el propio Reglamento sobre la Transparencia de la CNUDMI, establecen una regulación más amplia de la institución al permitir que el tribunal pueda valorar si existe algún interés público en el caso para admitir la participación del amicus curiae. Esto demuestra una asimetría a nivel internacional respecto al tratamiento del amicus curiae en el procedimiento arbitral. Ante tal desequilibrio, se hace necesaria la búsqueda de un consenso a nivel internacional que favorezca la creación de un estatus de amicus curiae en los procedimientos ISDS, a través del cual, puedan adquirir plenos derechos obligatorios, no discrecionales, de participación en el procedimiento. Que les permita no solo participar del procedimiento, sino también, exigir una mayor divulgación de este, ya que la presencia del amicus curiae en el procedimiento no siempre conlleva a una mayor divulgación o conocimiento público del procedimiento y de todo cuanto sucede en el mismo ${ }^{32}$.

31 PETERSMANN, E.U., "Justice as Conflict Resolution: Proliferation, Fragmentation, and Decentralization of Dispute Settlement in International Trade", U.PA.J. Int'l. Econ.L., Vol. 27, 2006, pp. 273-280.

32 PETERSON, L., "Amicus Curiae Interventions: The Tail That Wags the Transparency Dog”, Kluwer Arb. Blog, 2010. Disponible en: http://kluwerarbitrationblog.com/2010/04/27/amicus-curiae-interventions-the-tail-that-wags-thetransparency-dog/, consultado 15/07/2020. 
El amicus curiae debe ser tratado como una valiosa institución que puede incidir en la búsqueda de una mayor armonización y coherencia en la interpretación de los TBI en los que se basan el ISDS. En este sentido, favorece a la existencia de una estrecha relación entre transparencia, coherencia —entendida esta como "consistencia de la regla y su aplicación con otras reglas"33 - y legitimidad en el procedimiento arbitral ${ }^{34}$. De esta manera, se puede aspirar a satisfacer los altos estándares de transparencia exigibles en el arbitraje de inversiones, una mayor credibilidad pública del proceso, así como una mayor legitimidad procesal y sustantiva del ISDS.

Creemos que las actuales propuestas de enmiendas presentadas por el CIADI van en camino de considerar la importancia y relevancia que tiene la participación de un tercero no contendiente en el procedimiento. En este sentido, el CIADI, aunque las modificaciones propuestas en el tema no son verdaderamente profundas, al mantenerse prácticamente iguales, introduce como novedad dos criterios que deben ser considerados para permitir la participación de terceros no contendientes en el procedimiento, a saber la identificación de las actividades del tercero y cualquier afiliación con una parte contendiente ${ }^{35}$ y si el tercero no contendiente ha recibido asistencia financiera o de cualquier otro tipo para realizar la presentación de su escrito ${ }^{36}$.

Además, las actuales propuestas le otorgan al tribunal discrecionalidad para que le ordene al tercero contribuir con el aumento de costos imputable a su participación ${ }^{37}$. Una propuesta muy interesante y que, si bien habrá que esperar su definitiva aprobación,

33 BRUNNEE, J., TOOPE, S.J., Legitimacy and Legality in International Law: An Interactional Account, Cambridge University Press, Cambridge, 2010, p. 53.

34 RUSCALLA, G., "Transparency in International Arbitration: Any (Concrete) Need to Codify the Standard?” GroJIL, Vol. 3, No. 1, 2015, p. 4.

35 Propuesta de Regla 48(2d) del Reglamento de Arbitraje CIADI: "la identidad, actividades, organización y los propietarios de la parte no contendiente, lo cual incluye toda afiliación directa o indirecta entre la parte no contendiente, una parte o una parte no contendiente del tratado".

36 Propuesta de Regla 48(2e) del Reglamento de Arbitraje CIADI: "si alguna persona o entidad le proporcionara a la parte no contendiente asistencia financiera $u$ otro tipo de asistencia para efectuar la presentación".

37 Propuesta de Regla 48(4) del Reglamento de Arbitraje del CIADI: "El Tribunal deberá asegurarse de que la participación de la parte no contendiente no perturbe el procedimiento, o genere una carga indebida, o perjudique injustamente a cualquiera de las partes. A tal fin, el Tribunal podrá imponer condiciones a la parte no contendiente, lo cual incluye con respecto a lo siguiente". En relación con la propuesta de Regla 57 del Reglamento del Mecanismo Complementario. 
estamos seguros de que no será de fácil implementación y aplicación en la práctica.

\section{LA FINANCIACIÓN POR TERCEROS EN EL ARBITRAJE INTERNACIONAL}

La financiación por terceros —conocida en términos ingleses como Third Party Funding (TPF)- y la transparencia se han convertido en temas cruciales y de gran significación en el arbitraje internacional ${ }^{38}$. El encarecimiento de los procedimientos de arbitraje internacional ${ }^{39}$ para las partes en litigio es uno de los motivos que ha favorecido la existencia reciente del tercero financiador, con mayor énfasis en el arbitraje de inversiones ${ }^{40}$, teniendo en cuenta las peculiaridades del arbitraje.

38 SCHERER M., GOLDSMITH A., "Third Party Funding in International Arbitration in Europe: Part 1-Funders Perspectives", Revue de Droit des Affaires Internationales/International Business Law Journal, No. 2, 2012, pp. 207-210.

39 Lo que reafirma también, CREMADES, al expresar: “(...) sin embargo, la estructura internacional construida alrededor del arbitraje se ha vuelto costoso. El arbitraje se ha convertido en una gran industria internacional, enormemente competitiva y extremadamente costosa". CREMADES, B.M., "Third party funding in international arbitration", B. Cremades y Asociados, 2011, p. 2. Disponible en: https://www.cremades. com/pics/contenido/File634523783352588756.pdf, consultado el 15/07/2020; SEVILA, E., ANDRÉS O., "La financiación por terceros en el arbitraje internacional: regulación, ventajas e inconvenientes", Actualidad Jurídica Aranzadi, No. 941, 2018, p. 11.

40 DE BRABANDERE, E., LEPELTAK, J., "Third Party Funding in International Investment Arbitration", Grotius Centre for International Legal Studies, No.1, 2012, p.3. Creemos necesario mencionar, sin adentrarnos en el análisis de las mismas, ya que nos distanciaríamos del objeto de estudio de la presente investigación, que en el sistema anglosajón, la práctica del tercero financiador ya tenía desde mucho antes su espacio, con la doctrina "of champerty, barratry and maintenance", aunque con una connotación distinta, desde el ámbito de los litigios civiles. Véase; CREMADES, B.M.: Third party funding in international arbitration....op.cit...nota 37, p. 4; FLAKE, C.R., "Third Party funding in domestic arbitration: champerty or social utility?", Dispute Resolution Journal, Vol. 70, No. 2, 2012, pp.110-114; NG, J.F., "The Role of the Doctrines of Champerty and Maintenance in Arbitration", Arbitration. Vol. 76, 2010, pp. 208-209; DE BOULLE, T., Third-Party funding in international commercial arbitration, Ghent University, 2014, pp. 40-47; LYON, J., "Revolution in Progress: Third-Party Funding of American Litigation", UCLA. Law Review, Vol. 58, 2010, pp. 579-587; TRUSZ, J.A., "Full Disclosure? Conflicts of Interest Arising from Third-Party Funding in International Commercial Arbitration", Georgetown Law Journal, Vol. 101, No. 6, 2013, pp. 1659-1661. STEINITZ, M., "Whose Claim Is It Anyway? Third-Party Litigation Funding", Minnesota Law Review, Vol. 95, 2011, pp. 1286-1291; CITING MULHERON, R., CASHMAN, P., "Third Party Funding- A Changing Landscape", Civil Justice Quarterly, No. 3, 2008, pp. 312-341; BARKER, G.R., "Third Party Funding in Australia and Europe", Law and Economics Center, 2011; SHEPHERD, J.M., "Ideal versus reality in third-party litigation financing", Journal Law, Economics \& Policy, Vol. 8, No. 3 2012, pp.593-604; GLICKMAN, D.R., 
No puede negarse que independientemente de los riesgos existentes en la financiación por terceros, los cuales pueden estar, además, muy estrechamente relacionados con los significativos costes del proceso arbitral ${ }^{41}$, la financiación por terceros en el arbitraje internacional también tiene sus ventajas, resultando beneficioso, en gran medida, a la parte financiada. En primer lugar, la parte financiada tendrá la posibilidad de acceder al proceso arbitral, que de otra manera le sería imposible por no contar con los medios financieros necesarios $^{42}$. En segundo lugar, puede que la parte financiada, contando con los medios financieros necesarios, no desee hacer uso de sus recursos para encarar un proceso arbitral, teniendo en cuenta los altos costos, la duración y la posible influencia que pueda tener en su economía y finanzas ${ }^{43}$, por lo que puede beneficiarse de invertir en otros campos y obtener la financiación de un tercero financiador $^{44}$. Y, en tercer lugar, la parte financiada no solo contará con una provisión de fondos de financiación para demandar o afrontar un proceso arbitral, también puede llevar a cabo una transmisión de todos los riesgos - o parte de ellos - a asumir durante el procedimiento ${ }^{45}$ al financiador. Esto favorece a una externalización de los riesgos financieros asociados al procedimiento arbitral. Lógicamente, en caso de obtenerse una resolución favorable del proceso, la parte financiada obtendrá beneficios sin haber asumido grandes riesgos ${ }^{46}$, incluyendo los costes del propio proceso.

Sin embargo, actualmente no existe acuerdo a nivel internacional a través del cual se pueda conocer las características exactas de la financiación por terceros ${ }^{47} \mathrm{y}$, en consecuencia, un concepto único

"Embracing third-party litigation finance", Florida State University Law Review, Vol. 43, 2016, pp. 1052-1057; SEBOK, A.J., "Betting on Tort Suits after the Event: From Champerty to Insurance, DePaul Law Review, Vol. 60, No. 453, 2012, pp. 2011-2041.

41 BERT, I.H., "Litigation Finance: What Do Judges Need to Know?", Colum. J.L. \& Soc. Probs, Vol. 45, 2012, p. 527; KIDD, J., "To Fund or Not to Fund: The Need for Second-Best Solutions to the Litigation Finance Dilemma", Journal Law, Economics \& Policy, Vol. 8, 2012, pp. 634-635.

42 DE MOT, J., FAURE, M.G., VISSCHER. L., "TPF and Its Alternatives: An Economic Appraisal", Research Conference on Third Party Funding of Litigation, Leiden Law School, 2016, p. 1.

43 STEINITZ, M.: Whose Claim Is It Anyway...op.cit...nota 38, p. 1267.

44 DE BRABANDERE, E., LEPELTAK, J., Third Party Funding ....op.cit...nota 38 , p. 3.

45 FLAKE, C.R.: Third Party funding in domestic arbitration...op.cit...nota 38, pp.115-116.

46 MANN, H., "Reconceptualizing international investment law: Its role in sustainable development", Lewis \& Clark Law Review, Vol. 17, No. 2, 2013, p. 534.

47 Algunos autores, reconociendo la naturaleza exacta del financiamiento de tercero, han considerado la necesidad de encontrar actividades que tengan similitud 
del mismo. La financiación por terceros es una tendencia entre empresas con un amplio espectro transnacional, teniendo en cuenta que los terceros financiadores, cada vez más, invierten en litigios fuera de sus fronteras nacionales. Esto ha permitido una multitud de definiciones o criterios por quienes se han acercado al estudio del tema $^{48}$, favoreciendo, en gran medida, el inexplorado conocimiento de sus implicaciones éticas y jurídicas en el arbitraje internacional. En consecuencia, ante la imposibilidad de una definición única de la financiación por terceros, le corresponde a la comunidad jurídica internacional en lo general, y en particular a la comunidad arbitral, ir construyendo el camino que nos permita llegar a un concepto único, a partir de las distintas variantes en que se puede manifestar la financiación por terceros ${ }^{49}$.

En relación con lo anterior, y como punto de partida que nos permita un mejor desarrollo y análisis de la cuestión objeto del presente epígrafe, somos del criterio de que el tercero financiador, es aquel tercero ajeno a la controversia que lleva a cabo la financiación de los costos del arbitraje de una de las partes contendientes (generalmente, financia a los demandantes ${ }^{50}$ ), acordándose entre ellos la remuneración que recibirá el tercero financiador por la financiación

con la financiación de terceros y que puedan ofrecen una mejor visión o al menos encontrar puntos de referencias en común, teniendo en cuenta que la financiación por terceros puede ser considerada como un contrato de préstamo; una forma de seguro; un financiamiento de capital de riesgo, o; un acuerdo alternativo de honorarios abogado-cliente. SEBOK A.J, BRADLEY WENDEL W., "Characterizing the Parties' Relationship in Litigation Investment: Contract and Tort Good Faith Norms", Vanderbilt Law Review, Vol. 66, No. 1, 2013, pp. 1832-1833.

48 La inexistencia de un concepto de financiación de terceros, ha propiciado que la actividad sea definida o conceptualizada por algunos autores desde varias perspectivas, como puede ser: desde lo económico, lo financiero y lo jurídico, FLÉCHET C., "Summary report of the first session held", Revue de Droit des Affaires Internationales/International Business Law Journal, No. 2, 2012, p. 209. En este mismo sentido, SCHERER, lo confirma correctamente cuando expresa que "la definición exacta de financiación de terceros, sin embargo, sigue siendo esquiva y sus implicaciones legales y éticas en el arbitraje internacional, en su mayoría inexploradas"; SCHERER, M., "Third-party funding in international arbitration: Towards mandatory disclosure of funding agreements?", en CREMADES, B., DIMOLITSA, A. (Eds.): Dossier X: Third-party Funding in International Arbitration, ICC Publishing S.A., Paris, 2013, p. 95. Puede verse también, algunas definiciones al respecto en, DE BOULLE, T., Third-Party funding in international commercial arbitration..., op.cit..nota 38, pp. 5-6.

49 CREMADES, B., "Concluding remarks", en CREMADES, B., DIMOLITSA A. (Eds.): Dossier X: Third-party Funding in International Arbitration, ICC Publishing S.A., Paris, 2013, p. 153.

50 DERAINS Y., "Foreword", en CREMADES B.M., DIMOLITSA A (Eds.): Dossier X: Third-party Funding in International Arbitration, ICC Publishing S.A., París, 2013, p. 5. 
de todo el proceso o parte del mismo, predominando más en los procedimientos de arbitraje de inversiones que en el arbitraje comercial internacional ${ }^{51}$. Es decir, en el arbitraje internacional la financiación por terceros se establece mediante un acuerdo o contrato de financiación entre un financiador comercial - generalmente una empresa o un fondo de inversión con amplia experiencia profesional-que acepta el pago de los honorarios y los gastos legales ${ }^{52}$ para hacer frente a todo o parte del proceso, a cambio de la devolución de todos los gastos incurridos por el financiador, más una parte de lo obtenido en la reclamación del caso. El tercero financiador no tiene interés en las cuestiones sustantivas del caso, su principal interés es invertir financieramente para hacer frente de los gastos del procedimiento de una de las partes ${ }^{53}$.

Ahora bien, en caso de que la parte contendiente que ha recibido el financiamiento resulte vencedora en el proceso, no debe existir inconveniente alguno para efectuar el pago de la remuneración al financiador, ya que la suma obtenida por la parte financiada será de una cantidad considerable, teniendo en cuenta los altos costos del proceso y, además, las altas indemnizaciones a pagar en las

51 La decisión de un financiador respecto a la aceptación de financiar a una de las Partes contendientes todo el proceso arbitral o parte de este no resulta fácil, existiendo como uno de los principales problemas en el ámbito del arbitraje internacional, que la gran mayoría de la actividad financiera en la actualidad está relacionada con disputas domésticas. Véase, SEIDEL S., "Third-party investing in international arbitration claims To invest or not to invest? A daunting question", en CREMADES B.M., DIMOLITSA A (Eds.), Third-party Funding in International Arbitration, ICC Publishing S.A., París, 2013, p. 16.

52 KHOURI, S., HURFORD, K., "Third party funding for international arbitration claims: practical tips", Practical Law Company Arbitration, 2014, p. 2. Disponible en: www.practicallaw.com, consultado el 13/05/2020; KHOURI, S., HURFORD, K., BOWMAN, C., "Third party funding in international commercial and treaty arbitration - A panacea or a plague? A discussion of the risks and benefits of third party funding", Transnational Dispute Management, Vol. 8, No.4, 2011, p. 3.

53 VAN BOOM, W.H., "Financing civil litigation by the European insurance industry", en TUIL, M., VISSCHER, L. (Ed.): New trends in financing civil litigation in Europa: a legal, empirical, and economic analysis, Cheltenham/Northampton: Edward Elgar, 2010, pp. 92-108; FERNÁNDEZ MASIÁ, E., "La financiación por terceros en el arbitraje internacional", Cuadernos de Derecho Transnacional, Vol. 8, No. 2, 2016, pp. 206-207. En la práctica existen también otros tipos de terceros financiadores - que, si bien no son objeto de estudios en el presente apartado, consideramos necesario mencionar- los cuales apoyan financieramente a una de las partes en el proceso arbitral, pero sin recibir nada a cambio, o sea, sin interés en recibir una compensación por el apoyo financiero aportado y que suelen ser generalmente fundaciones. Cuestión esta que algunos autores consideran como donaciones y no un acuerdo de financiación en sí, teniendo en cuenta los riesgos inherentes en éste último. DE BRABANDERE, E., LEPELTAK, J., Third Party Funding....op.cit.., nota 38., p. 6. 
demandas. Cuestión distinta resulta cuando la parte financiada viese desestimada sus pretensiones. Al respecto, el tercero financiador asumirá el riesgo de toda la pérdida ${ }^{54}$, incluyendo otros costos que se haya obligado a asumir generados durante el plazo de vigencia del acuerdo de financiación, sin posibilidad de exigir a la parte financiada compensación de ninguna clase.

No hay duda de que el número de arbitrajes internacionales aumenta $^{55}$ a un ritmo directamente proporcional al crecimiento que en los últimos años ha ido experimentando la propia actividad de financiación por terceros ${ }^{56}$. Los financiadores investigan y se interesan,

54 KHOURI, S., HURFORD, K., BOWMAN, C., Third party funding in international commercial...op.cit...nota 50, p. 3; FERNÁNDEZ MASIÁ, E., La financiación por terceros en el arbitraje internacional...op.cit..nota 51, p. 207; NAVARRO, S., "Cuestiones relativas al third party funding en arbitraje", Arbitraje. Revista de arbitraje comercial y de inversiones, Vol.VII, No. 2, 2014, p.803. En relación, a su vez, con la conceptualización que le ha dado JACKSON en el glosario del Informe Final sobre la revisión de los costos en la litigación civil, JACKSON, R., Review of Civil Litigation Costs: Final Report, TSO Ireland, 2009, p. viii; DESTEFANO, M., "Nonlawyers Influencing Lawyers: Too Many Cooks in the Kitchen or Stone Soup?", Fordham L. Rev, Vol. 80, 2012, p. 2818; VELJANOVSKI, C., "Third-Party Litigation Funding in Europe", Journal Law, Economics \& Policy, Vol. 8, No. 3, 2012, p. 3; También, la consulta del Gobierno de Hong Kong, "Third Party Funding for arbitration, the law reform commission of Hong Kong, p. 4. Disponible en: http://www. hkreform.gov.hk, consultado el 14/05/2020.

55 SANTOSUOSSO, T., SCARLETT, R., "Third-Party Funding in Investment Arbitration: Misappropriation of Access to Justice Rhetoric by Global Speculative Finance", Law and Justice in the Americas Working Paper Series, No. 8, 2018, p. 5. Disponible en: http://lawdigitalcommons.bc.edu/ljawps/8, consultado el 14/05/2020; DOS SANTOS, C., "Third-party funding in international comercial arbitration: a wolf in sheep's clothing?", ASA Bulletin, Vol. 35, No. 4, 2017, p. 918; DE BOULLE, T., Third-Party funding in international commercial arbitration, .....op.cit...nota 38, p. 1.

56 Son muchos los casos que demuestran el crecimiento de la participación de terceros en el arbitraje internacional, entre los cuales podemos citar: Bernardus Henricus Funnekotter and others vs. Republic of Zimbabwe, ICSID Case No. ARB/05/6, 2003, resuelto en 2009. Disponible en: https:/www.italaw.com/documents/ZimbabweAward.pdf, consultado el 14/05/2020; Gustav F W Hamester GmbH \& Co KG vs. Republic of Ghana, ICSID Case No. ARB/07/24, 2010. Disponible en: https:/www.italaw.com/sites/default/files/case-documents/ita0396.pdf, consultado el 14/05/2020; Chevron Corporation (USA) and Texaco Petroleum Company (USA) vs. The Republic of Ecuador, UNCITRAL, PCA Case No. 34877, 2008. Disponible en: https://www.italaw.com/cases/251, consultado el 14/05/2020; Waguih Elie George Siag and Clorinda Vecchi vs. The Arab Republic of Egypt, ICSID Case No. ARB/05/15, 2005. Disponible en: https://www.italaw.com/cases/1022, consultado el 14/05/2020. Véase también, BURKE ROBERTSON, C., "The Impact of Third-Party Financing on Transnational Litigation", Case Western Reserve Journal of International Law, Vol. 44, 2012, pp.168-169; BENCH NIEUWVELD, L., SHANNON, V., "Third-Party Funding in International Arbitration", Kluwer Law International, 2012, p. 3; SEIDEL, S., "Fulbrook Primer on Third Party Dispute Finance", Fulbrook Capital Management, 
cada vez más, en la búsqueda de nuevos casos con el ánimo de financiar los litigios arbitrales ${ }^{57}$. El aumento de la financiación por terceros resulta más notorio en algunas jurisdicciones como pueden ser Estados Unidos, Reino Unido y Australia ${ }^{58}$. Esto constituye un gran desafío, no solo para la parte contendiente que necesita del apoyo financiero para poder emprender un proceso arbitral o compartir los riesgos a asumir durante el proceso, sino también para los propios financiadores, que deben estar al tanto de las principales actualizaciones en el ámbito del arbitraje internacional, analizar, conocer, las reglas que los rigen y las experiencias que puedan tener según los diferentes casos. De ahí, la importancia de realizar un pormenorizado

LL.C, 2016, pp. 23-25; LYON, J., Revolution in Progress....op.cit...nota 38, p. 571; YU, H.L., "Can third party funding deliver justice in international commercial arbitration?", International Arbitration Law Review, Vol. 20, No. 1, 2017, pp. 21-22. El aumento de la financiación por terceros es también corroborado, en el borrador del informe ICCA-Queen Mary Task Force on Third-Party Funding In International Arbitration, 2017, p.1. Disponible en: http://www.arbitration-icca.org/projects/Third_ Party_Funding.html, consultado el 14/05/2020; HONLET, J.C., Recent decisions on third-party funding in investment arbitration", ICSID Review, 2015, pp. 699-712.

57 Cuestión que, coincidiendo con SEIDEL, se ve alentada por varios factores como son: el aumento del comercio; mayor competencia entre los países para convertirse en sedes arbitrales y que ven la financiación por terceros como una ventaja comercial, y; mayor experiencia en el ámbito internacional del arbitraje internacional y la financiación por terceros, SEIDEL S., Third-party investing in international arbitration...op.cit...nota 49, pp. 16-17.

58 En dichas jurisdicciones, la financiación por terceros está muy presente en litigios judiciales y en ámbitos tan específicos como el Derecho de la competencia. No obstante, aunque existen pocos datos estadísticos que demuestren el aumento de la actividad de financiación por terceros en el arbitraje internacional, teniendo en cuenta el carácter confidencial del mismo, la evidencia práctica demuestra que dicha actividad va cada vez más en aumento. Véase, KHOURI, S., HURFORD, K., Third party funding for international arbitration...op cit..nota 50, p. 3; MILES, C., ZAGATA VASANI, S., "Case Notes on Third-Party Funding", Global Arbitration Review, Vol. 3, No. 1, 2008, p. 35. El motor impulsor respecto a la regulación de la financiación por terceros fue el Reino Unido, a través del Code of Conduct for Litigation Funders, 2011. En Estados Unidos, la American Bar Association creó el denominado White Paper About Alternative Litigation Financing, en la que se establecen normas que deben cumplir los abogados estadounidenses cuando representan legalmente un caso que se encuentre financiado por un tercero, teniendo en cuenta que en la gran mayoría de los estados no está plenamente regulada la financiación por terceros. Aunque las propuestas más recientes van encaminadas, fundamentalmente, a la financiación por terceros en el ámbito de la protección al consumidor. En el caso australiano, es posible que sea la jurisdicción más favorable a nivel internacional a la financiación por terceros, contando con regulaciones y tribunales con gran experiencia al respecto. Tanto es así, que muchos consideran que el propio avance que tiene la legislación australiana respecto a la financiación de litigios por terceros, puede ser extrapolado al arbitraje. Véase, SHANNON, V., "Recent developments in Third-Party Funding", Journal of International Arbitration, 2013, p. 444; BENCH NIEUWVELD, L., SHANNON, V., Third-Party Funding in International Arbitration...op.cit...nota 54, pp. 71-94 
estudio, teniendo en cuenta la complejidad de los litigios objeto de arbitrajes y la cambiante e inestable situación económica y financiera a nivel global. Sin embargo, la metodología empleada por las empresas financieras para evaluar exhaustivamente todo el proceso de financiamiento de una parte en litigio es poco conocida $^{59}$.

Como bien hemos comentado antes, en el ámbito del arbitraje internacional, todo procedimiento arbitral es sumamente costoso ${ }^{60}$. Pero el ISDS bajo tratados de inversión, tienden a ser más onerosos que el arbitraje comercial internacional ${ }^{61}$. La actividad inversora de la que se ha originado el litigio objeto del procedimiento arbitral ha propiciado en sí una inversión sumamente beneficiosa y rentable para el tercero financiador ${ }^{62}$. El rol de un tercero financiador en el procedimiento arbitral es muy amplio, puede ir desde el simple préstamo de dinero, sin compartir los riesgos del proceso, hasta involucrarse totalmente en el proceso, como un inversor más. La decisión de un financiador a financiar un proceso arbitral puede constituir, en sí misma, una inversión, convirtiéndose básicamente el tercero financiador en un inversor más del procedimiento arbitral ${ }^{63}$.

Es por ello, que más allá de la evaluación de los riesgos financieros y la valoración de todos los aspectos de la reclamación que se le exige asumir o compartir ${ }^{64}$ al tercero financiador, debe tenerse en cuenta el resto de los factores comentados antes y que se encuentran muy relacionados con la propia actividad arbitral. Al respecto, debe tenerse en cuenta que el arbitraje no constituye una actividad

59 CREMADES JR, B.M., "Third Party Litigation Funding: Investing in Arbitration”, Transnational Dispute Management, Vol. 8, No.1, 2011, pp. 12-15.

60 UNCTAD, Investor-State Disputes: Prevention and Alternatives to Arbitration, New York/Geneva, 2010, p. 17; BORN, G.B, International commercial arbitration, Wolters Kluwer Law \& Business, Primera Edición, The Netherlands, 2009, p. 2488.

61 PARK, W.W., ROGERS, C.R., "Third-Party Funding in International Arbitration: The ICCA Queen-Mary Task Force”, 2014, p. 9. Disponible en: http://ssrn.com/ abstract $=2507461$, consultado el 14/05/2020.

62 DE BRABANDERE, E., LEPELTAK, J., Third Party Funding in International Investment Arbitration...op.cit...nota 38, p. 9.

${ }_{63}$ En este sentido, VAN BOOM, reconoce que en términos económicos, la participación de un tercero como inversor del proceso arbitral “(...) podría clasificarse como un instrumento de deuda pura, un instrumento de prevención de riesgos, una inversión compartida basada en acciones o una simple venta del reclamo". Véase, VAN BOOM W.H., "Third-Party Financing in International Investment Arbitration", $O E C D, 2011$, p. 27; MANIRUZZAMAN, M., "Third-party funding in international arbitration - A menace or panacea?", Kluwer Arbitration, No. 29, 2012, p.1. Disponible en: http://kluwerarbitrationblog.com/blog/2012/12/29/third-party-funding-in-international-arbitration-a-menace-or-panacea/, consultado el 14/06/2020.

${ }_{64}$ KHOURI, S., HURFORD, K., Third party funding for international arbitration...op cit..nota 50, pp.7-8. 
propia del financiador, le resulta totalmente ajeno a su objeto empresarial. Dicho esto, el financiador necesita como requisito sine qua non, un minucioso análisis de las especificidades del proceso arbitral, incluyendo, entre otros, el propio acuerdo arbitral entre las partes contendientes, la posibilidad de ejecución del laudo, las posibilidades reales de obtener la compensación y el tiempo estimado para obtener una resolución del proceso. De ahí la importancia de la existencia de normas de transparencia que puedan facilitar dicho conocimiento.

\section{III.1. El acuerdo de financiación con terceros y su influencia en el procedimiento arbitral ¿Está sujeto el acuerdo de financiación a normas de transparencia y a obligación de divulgación?}

Como decíamos la participación de terceros financiadores en el arbitraje internacional ha ido creciendo en los últimos años ${ }^{65}$. A pesar de dicho aumento, el conocimiento y divulgación en el procedimiento arbitral de la presencia del tercero financiador y su relación con la parte financiada, específicamente sobre el acuerdo arbitral, sigue siendo una incógnita en la mayoría de los $\operatorname{casos}^{66}$.

Tienen gran trascedencia las normas de transparencia en el arbitraje internacional, en el acuerdo de financiación entre la parte financiada y el tercero financiador en el procedimiento arbitral. En este sentido, la revelación del acuerdo de financiación y la presencia del tercero financiador se han convertido en temas ferozmente discutidos, debido a la importancia y preeminencia que tienen en el procedimiento arbitral. Por esta razón ha de preguntarse si existe la obligación de poner en conocimiento del tribunal y de terceros el

65 A pesar de la ausencia de estadísticas que evidencien el aumento en la participación de terceros en el arbitraje comercial internacional, teniendo en cuenta el carácter confidencial del mismo. En el arbitraje de inversiones no ha resultado igual, debido al carácter cuasipúblico de este tipo de arbitrajes, en la práctica se ha demostrado en multitud de casos la presencia de un tercero financiador. Véase, TRUSZ, J.A., Full Disclosure...op.cit...nota 38, pp. 1651, 1665; HARWOOD, M.K., BATIFORT, S.N., TRAHANAS, CH., "Third-Party Funding: security for costs and other key issues", en LEGUM, B. (Ed.), The Investment Treaty Arbitration Review, Law Business Research, Second Edition, 2017, p. 104.

66 En este sentido, STEINITZ, reconoce que el tercero financiador trata toda inversión como su propiedad, lo que motiva que la inversión que realizan en un procedimiento arbitral, incluyendo el propio acuerdo arbitral, resulta ser secreto. Véase, STEINITZ, M., "The Litigation Finance Contract", William \& Mary Law Review, Vol. 54, 2012, pp. 455, 463. 
acuerdo de financiación. Dicha obligación resultaría difícil implementar, aunque no imposible, teniendo en cuenta, entre otros inconvenientes, la falta de definición del concepto de tercero financiador y lo que se establecerá en el ámbito de tal obligación. Cuestiones que analizamos en lo adelante.

III.1.A. La transparencia en el arbitraje y el acuerdo de financiación con terceros: el deber de revelación del acuerdo de financiación.

En nuestra opinión, teniendo en cuenta las particularidades del acuerdo de financiación, el cual es único y específico para cada litigio en concreto, y constituye la piedra angular de la financiación. Consideramos que resulta imprescindible la existencia de normas que permitan su conocimiento por todas las partes en el proceso ${ }^{67}$, incluyendo terceras personas interesadas. En concreto, las razones para ello son las siguientes: primero, se evita la existencia de posibles conflictos de intereses por los árbitros; en segundo lugar, por motivos de transparencia procesal e identificar la verdadera parte en el caso para que, de resultar posible y en caso de ser solicitado, el tribunal arbitral pueda ordenar una caución o cautio judicatum solvi (security for costs) como garantía para el demandando, a la que nos referiremos más adelante. En este sentido, el tribunal puede tener un mayor conocimiento del acuerdo y si el mismo ha sido realizado justa y razonablemente para los fines establecidos, teniendo en cuenta los riesgos asociados al arbitraje y las circunstancias de la financiación.

67 La divulgación del acuerdo de financiación ha sido muy controvertida a nivel académico y en la práctica. Ante quienes defendemos la divulgación del acuerdo de financiación en su totalidad, existen otros que prefieren divulgar solo una parte, alegando que la divulgación total del acuerdo puede ser tenido en cuenta por el tribunal arbitral en sus decisiones. Véase, APPELBAUM, B., "Lawsuit Loans Add New Risk for the Injured", The New York Times, 2011. Disponible en: www.nytimes.com/2011/01/17/business/17lawsuit.html?pagewanted=all\&_r=0, consultado el 14/06/2020; MARTIN, S., "The Litigation Financing Industry: The Wild West of Finance Should Be Tamed Not Outlawed", Fordham Journal of Corporate \& Financial Law, Vol. 10, 2004, p. 55. El fundamento de esta divulgación se explica por las numerosas y diversas cuestiones legales que están relacionadas y afectan directa e igualmente la cuestión de la independencia del árbitro, así como la incidencia en los costos. Véase, NASR, I., Third-Party funding in international arbitration, University of Carthage, 2015, p. 64. Aunque otros autores defienden que debe existir causas o condiciones inusuales durante el proceso para poder exigir tal obligación. LEVY, L., BONNAN, R., "Third-party funding: Disclosure, joinder and impact on arbitral proceedings “, en CREMADES, B.M., DIMOLITSA, A. (eds.): Dossier X: Thirdparty Funding in International Arbitration, ICC Publishing S.A., Paris, 2013, p. 78. 
Además, como se dijo, hay que tener en cuenta que la mayor presencia de terceros financiadores se encuentra en el ISDS ${ }^{68}$. La concurrencia de un Estado soberano, responsable ante sus ciudadanos de las actuaciones que realiza, incluyendo la propia responsabilidad que asume ante el arbitraje, justifican la revelación del tercero financiador y la divulgación del acuerdo de financiación. El caso Muhammet Cap \& Sehil Insaat Endustri ve Ticaret Ltd Sti vs. Turkmenistan ${ }^{69}$ es el reflejo claro de la obligación de divulgación del acuerdo de financiación en el arbitraje de inversiones. Los hechos del presente caso se relacionan con el incumplimiento de pago de la demandada (Turkmenistan) de varios proyectos de construcción realizados por los demandantes (Insaat Endustri ve Ticaret Ltd Sti), así como la expropiación de la inversión sin compensación alguna. Durante el desarrollo del procedimiento arbitral, en el año 2014, la demandada solicitó al tribunal que ordenara a los demandantes divulgar, entre otras cuestiones, si habían firmado un acuerdo de financiación con terceros para financiar sus reclamaciones $\mathrm{y}$, de resultar positivo, que revelaran los términos de dicho acuerdo ${ }^{70}$. Solicitud que inicialmente fue rechazada por el tribunal al entender que no existían motivos para la emisión de tal orden.

Sin embargo, en el año 2015 la demandada solicitó nuevamente al tribunal que emitiera una orden que revelara la identidad y naturaleza de la participación de un tercero financiador en el procedimiento, teniendo en cuenta que, en la anterior solicitud, si bien los demandantes se opusieron a la misma, no negaron la posible existencia de un tercero financiador. Además, la solicitud tuvo como motivo principal que los demandantes cambiaron de asesores legales, pasando a ser representados por los mismos que

68 El arbitraje de inversiones se ha convertido en el epicentro de la financiación por terceros, muchos casos así lo reafirman, encontrándose entre otros: Ron Fuchs vs. The Republic of Georgia, ICSID Case No. ARB/07/15, 2007, financiado por Allianz Litigation Funding. Disponible en: http://investmentpolicyhub.unctad.org/ ISDS/Details/268 y https://www.italaw.com/cases/464; Rusoro Mining Ltd. vs. Bolivarian Republic of Venezuela, ICSID Case No. ARB(AF)/12/5, 2012, financiado por Calunius Capital. Disponible en: https://www.italaw.com/cases/2048; S\&T Oil Equipment and Machinery Ltd. vs. Romania, ICSID Case No. ARB/07/13, 2007, financiado por Jurídica. Disponible en: http://investmentpolicyhub.unctad.org/ISDS/Details/277; Teinver S.A., Transportes de Cercanias S.A. and Autobuses Urbanos del Sur S.A. vs. The Argentine Republic, ICSID Case No. ARB/09/1, 2012, financiado por Burford. Disponible en: https://www.italaw.com/cases/1648; PARK, W.W., ROGERS, C.R., Third-Party Funding in International Arbitration...op.cit..., p. 3. Todos los sitios web fueron consultados 16/05/2020.

69 Muhammet Cap \& Sehil Insaat Endustri ve Ticaret Ltd Sti vs. Turkmenistan, ISCID Case No ARB/12/6, 2015, Procedural Order No 3.

70 Ibidem, Procedural Order No. 3, paragraph. 1. 
representaban, en otro caso distinto contra la propia demandada, un procedimiento de anulación. También alegó que era necesaria la revelación del tercero con el objetivo de prevenir la existencia de conflictos de intereses con las partes involucradas en el procedimiento, particularmente con los árbitros, y que valoraba, además, solicitar al tribunal que emitiera una orden de caución de las costas adversas, teniendo en cuenta que el tercero financiador podía retirarse del procedimiento en cualquier momento, evadiendo así cualquier posibilidad de asumir los costes adversos generados en el procedimiento ${ }^{71}$. Como era de esperar, los demandantes manifestaron su desacuerdo al respecto.

El tribunal, considerando la importancia que tiene la transparencia en casos como este para garantizar la integridad del procedimiento y la posible afectación (conflicto de interés) de alguno de los árbitros por la presencia del tercero financiador (énfasis propio) ${ }^{72} \mathrm{y}$, teniendo en cuenta la preocupación de la demandada que, de no ordenarse por el tribunal la constitución de una caución, en caso de obtener éxitos en el procedimiento, se vería imposibilitada de sufragar los gastos en los que ha incurrido durante el proceso, debido a que los demandantes no cuentan con la capacidad económica necesaria para sufragarlos y el tercero financiador habrá desaparecido teniendo en cuenta que no es parte del procedimiento ${ }^{73}$. Concluyó ordenándole al demandante que revelara el nombre y los detalles del acuerdo con el tercero financiador, la naturaleza del acuerdo de financiación, e incluso, hasta la posibilidad de éxitos que pudiese tener el tercero financiador en caso de que el demandante obtuviese una resolución favorable ${ }^{74}$.

71 Ibídem, paragraph. 2. "(...) that a third-party funder may elect to withdraw at any time and [...] may be able to evade a costs award in the event of an adverse decision".

72 Ibídem, paragraph 9. "First, the importance of ensuring the integrity of the proceedings and to determine whether any of the arbitrators are affected by the existence of a third-party funder. In this respect the Tribunal considers that transparency as to the existence of a third-party funder is important in cases like this".

73 Ibídem, paragraph. 12. "In the circumstances, the Tribunal is sympathetic to Respondent's concern that if it is successsful in this arbitration and a costs order is made in its favour, Claimants will be unable to meet these costs and the third-party funder will have disappeared as it is not a party to this arbitration".

74 Ibídem, paragraph 13. "Accordingly, the Tribunal has decided and hereby orders that within 15 days of the date of this Procedural Order, Claimants shall confirm to Respondent whether its claims in this arbitration are being funded by a third-party funder, and, if so, shall advise Respondent and the Tribunal of the name or names and details of the third-party funder(s), and the nature of the arrangements concluded with the third-party funder(s), including whether and to what extent it/they will share in any successes that Claimants may achieve in this arbitration". 
El ahora referido caso fue citado por los tribunales de los casos South American Silver Limited vs. Bolivia ${ }^{75}$, que consideró necesario la revelación del nombre del tercero financiador a efectos de la transparencia en el procedimiento, y Guaracachi America, Inc. and Rurelec PLC vs. The Plurinational State of Bolivia ${ }^{76}$, favoreciendo con la divulgación del tercero financiador que los árbitros se pronunciaran reconociendo la no existencia de conflictos de intereses y que, teniendo en cuenta el conocimiento previo de la presencia del tercero financiador, no habían motivos que perjudicaran la imparcialidad e independencia de los mismos.

La divulgación del tercero financiador puede facilitar el conocimiento respecto a la existencia de posible conflicto de interés ${ }^{77}$, con especial atención entre la representación legal —abogado- de la parte financiada y el tercero financiador y entre el tercero financiador con alguno de los árbitros, cuya existencia puede afectar el procedimiento arbitral en su totalidad ${ }^{78}$. Al respecto, se hace imprescindible la divulgación y el conocimiento del acuerdo de financiación, fruto de las negociaciones llevadas a cabo entre el financiador y la parte financiada. En este sentido, el abogado representante debe jugar un rol activo para que su cliente pueda tomar la más correcta decisión respecto a la financiación y tener pleno conocimiento de los riesgos que pueda asumir. Cuestión que resulta muy difícil cuando el abogado ha sido propuesto o designado por el propio financiador.

Un tema de máxima preocupación es el de la posible influencia que pueda recibir el abogado de la parte financiada por el tercero financiador, pudiendo existir cierto favoritismo de los intereses

75 South American Silver Limited vs. Bolivia, UNCITRAL, PCA Case No. 201315, Procedural Order No. 10, 2016, Paragraph 79. "Concerning the second point, the disclosure of the name of the funder, the Tribunal considers that, for purposes of transparency, and given the position of the Parties, it must accept Bolivia's request of disclosure of the name of SAS' funder".

76 Guaracachi America, Inc. and Rurelec PLC vs. The Plurinational State of Bolivia, UNCITRAL, PCA Case No. 2011-17, Procedural Order No. 13, 2013, Paragraph 9. "Por lo tanto, para evitar cualquier duda, los miembros del Tribunal manifiestan por la presente no tener ningún vínculo con Salvia Investment Limited, ni conocer de ninguna circunstancia que pudiera dar lugar a dudas justificadas acerca de su independencia o imparcialidad que pueda surgir de la financiación de los reclamos de las Demandantes por Salvia Investment Limited".

77 ROGERS, C.A., Ethics in International Arbitration, Oxford University Press, 2014, p. 201; SMITH M., "Mechanics of Third-Party Funding Agreements: A Funder's Perspective”, en AA.VV.: Third Party Funding in International Arbitration, Kluwer Law International, The Netherlands, 2012, p. 26.

78 SCHERER, M., Third Party Funding in International Arbitration...op.cit.. nota 46,, p. 210-219. 
de este último sobre los intereses de la parte financiada ${ }^{79}$, logrando motivar la existencia de un conflicto de interés para el abogado, teniendo en cuenta el deber de lealtad para con su cliente ${ }^{80}$. Motivos que también justifican el conocimiento del acuerdo de financiación por terceras partes, siempre que dichos terceros tengan alguna vinculación con el caso. Es por ello, que se necesita, sin lugar a duda, la existencia de reglas de transparencia que no solo puedan evitar la existencia de posibles conflictos de intereses, sino también, una mayor fiabilidad y claridad del acuerdo de financiación y de la participación del tercero financiador en el procedimiento arbitral en general. De ahí que, teniendo en cuenta que la financiación de terceros tiene mayor incidencia en el ISDS, resultando incuestionable el interés público presente en este tipo de $\operatorname{arbitrajes}^{81}$, resulta de gran utilidad que todas las partes involucradas en el caso, conozcan las particularidades del acuerdo de financiación, teniendo en cuenta su especificidad y complejidad.

En contraposición, existen algunos críticos que consideran necesario y suficiente revelar el acuerdo de financiación únicamente al tribunal arbitral ${ }^{82}$ y que dicha revelación no sea total, sino solamente

79 La posible relación o influencia que pueda recibir el abogado de la parte financiada es un tema muy delicado y de mucha preocupación en la práctica. Preocupación que ha sido expresada por académicos y estudiosos del tema, como lo demuestra RICHMOND, cuando expresa, "A primary concern with litigation funding is the potential for litigation funding companies to direct or regulate the professional judgment of the attorney with whom they deal, in order to protect the companies' investments. In so doing, the companies may impair the attorney's representation of his client. Courts commonly worry 'that an attorney's primary loyalty will, as a practical matter, rest with the person or entity who pays him.' Scholars share this concern"; RICHMOND, D.R., "Other People's Money: The Ethics of Litigation Funding," Mercer Law Review, Vol. 56, No. 2, 2005, pp. 649-682.

80 MCLAUGHLIN, J.H., "Litigation Funding: Charting a Legal and Ethical Course", Vermont Law Review, Vol. 31, 2007, p. 650.

81 La transparencia en el arbitraje de inversiones ha ido penetrando poco a poco en las profundidades del procedimiento arbitral, distanciándose en este aspecto del arbitraje comercial internacional. La presencia del Estado y la revisión de sus actos en el más pleno ejercicio soberano de sus funciones, amerita, sin lugar a dudas, la existencia de mayor transparencia procesal en el arbitraje inversorEstado. SCHILL, S.W., "Crafting the International Economic Order: The Public Function of Investment Treaty Arbitration and Its Significance for the Role of the Arbitrator", Leiden Journal of International Law, Vol. 23, No. 2, 2010, pp. 411- 415; DE BRABANDERE, E., "NGOs and the 'Public Interest': The Legality and Rationale of Amicus Curiae Interventions in International Economic and Investment Disputes", Chicago Journal of International Law, Vol. 12, 2011, pp. 85-113; VAN HARTEN, G., Investment Treaty Arbitration and Public Law, Oxford University Press, Oxford, 2007, pp. 48-49.

82 DE BOULLE, T., Third-Party funding in international commercial arbitration...op.cit...nota 38 , p. 83. 
una parte del acuerdo de financiación ${ }^{83}$, aduciendo que es el tribunal arbitral el máximo responsable de considerar las razones por las cuales divulgar el acuerdo de financiación. Creemos que este modo de proceder podría resultar razonable, si nos encontrásemos ante un arbitraje comercial internacional, exclusivamente entre partes privadas y sin presencia estatal, en el cual, el propio acuerdo arbitral permite a las partes contendientes ejercer mayor control del proceso. Pero no en el caso de un arbitraje de inversiones, en el que debe existir obligatoriamente un elevado grado de transparencia procesal, siendo en este régimen arbitral mucho más apremiante la divulgación del acuerdo de financiación por la fuerte presencia del interés público. No obstante, en ambos regímenes arbitrales, está obligado el tribunal arbitral a desempeñar sus funciones en correspondencia con la correcta administración de la justicia procesal en el arbitraje, asegurando así, la integridad del proceso ${ }^{84}$.

Sin adentrarnos en el análisis de a qué parte le corresponde la carga de la obligación de revelación del acuerdo, debido a que no está en consonancia con el objeto de estudio ${ }^{85}$. Creemos que el requisito de obligación debe aplicarse a todas las partes involucradas en el caso, asegurando siempre que dicha divulgación no perturbe el procedimiento arbitral. En este sentido, el arbitraje internacional será mucho más transparente, logrando una interacción entre todas las partes vinculadas al procedimiento, incluyendo el propio tribunal ${ }^{86}$.

No obstante, consideramos que puede existir parte de la información aportada en la consecución del acuerdo de financiación entre la parte financiada y el tercero financiador que no deba divulgarse o conocerse por terceras partes, como puede ser la información confidencial del financiador que no tenga directamente relación con el litigio y que, según la ley aplicable al caso, o la ley nacional del financiador, así lo establezca. En caso de omisión por estas, somos del criterio que debe ser una facultad exclusiva del tribunal decidir qué

83 MANIRUZZAMAN, M., Third-party funding in international arbitration... op.cit...nota 61, pp. 2-3.

${ }_{84}$ YU, H.L., Can third party funding...op.cit...nota 54, p 29.

85 Para interesados en conocer a quién le debe corresponder la carga de la obligación de revelación del acuerdo arbitral en el procedimiento, el autor recomienda verse a, TRUSZ, J.A., Full Disclosure....op.cit..nota 38, pp.1673-1674; SCHERER, M., GOLDSMITH, A., FLÉCHET, C., "Le financement par les tiers des procedures d'arbitrage international - une vue d'Europe Seconde partie: le debat juridique / Third Party Funding of International Arbitration Proceedings- A view from Europe Part II: The Legal Debate", Revue de Droit des Affaires Internationales/ International Business Law Journal, 2012, p. 653.

86 CREMADES, B.: Concluding remarks...op.cit...nota 47, p. 156. 
parte de la información puede o no ser confidencial, y no dejarla a la voluntad de las propias partes.

III.1.B) El deber de divulgación del acuerdo de financiación en las reglas arbitrales y tratados internacionales. Consideraciones desde el ámbito comparado.

En el ámbito de la financiación por terceros en el arbitraje internacional, independientemente de las particularidades del acuerdo de financiación entre las partes y la protección de la información confidencial que no tenga relación con el caso, lo más preocupante, y que a la vez, tiene una relación directa con la existencia de una mayor transparencia en este tipo de participación de terceros en el procedimiento arbitral, es que ninguna de las reglas institucionales: el CIADI $^{87}$, la Cámara de Comercio Internacional de París (CCI), así como las Reglas de Arbitrajes de la CNUDMI —incluyendo el propio Reglamento sobre la Transparencia de la CNUDMI-, contienen reglas específicas que regulen la financiación por terceros en el arbitraje internacional ${ }^{88}$.

Al no existir reglas institucionales que regulen dicha actividad, las propias instituciones arbitrales tienen como impedimento el poder tener un control directo, automático, respecto al conflicto de interés ${ }^{89}$. Al respecto, la financiación por terceros en el procedimiento arbitral queda a la libre disposición de las partes, bajo regulación de

87 Algunos autores, ante el vacío existente en las reglas institucionales respecto a la regulación de la financiación por terceros en el arbitraje internacional, reconocen que algunas instituciones ya están tomando partido en el asunto, por ejemplo: el CIADI. ANDRÉS ROS, O., SEVILA, E., "La financiación por terceros en el arbitraje internacional: regulación, ventajas e inconvenientes”, Andersen Tax \& Legal, 2018, p. 2.

88 TRUSZ, J.A., Full Disclosure...op.cit..nota 38, p. 1665; HARWOOD, M.K., BATIFORT, S.N., TRAHANAS, CH., "Third-Party Funding: security for costs and other key issues", en LEGUM, B. (Ed.), The Investment Treaty Arbitration Review, Law Business Research, 1st edition, 2016, p.97. Debemos resaltar, que las revisiones del 2014, de las reglas IBA a sus "Directrices sobre Conflictos de intereses en el arbitraje internacional", han intentado resolver en parte la situación, estableciendo en la norma general 6b, lo siguiente: "[i]f one of the parties is a legal entity, any legal or physical person having a controlling influence on the legal entity, or a direct economic interest in, or a duty to indemnify a party for, the award to be rendered in the arbitration, may be considered to bear the identity of such party". IBA Guidelines on Conflicts of Interest in International Arbitration. International Bar Association, 2014. Disponible en: http://www.ibanet.org, consultado el 14/06/2020.

89 El conflicto de interés ha sido muy reconocido por muchos estudiosos del tema, los cuales han considerado que la financiación por terceros en el arbitraje internacional, plantea "preocupaciones reales e importantes sobre conflictos potenciales"; 
sus leyes nacionales, la ley del lugar de la sede arbitral u otras leyes aplicables, situación que ha favorecido una mayor presencia de terceros financiadores en el arbitraje a nivel internacional, y con ello, un aumento en el número de casos de arbitraje de inversiones ${ }^{90}$. La práctica arbitral reafirma que la financiación por terceros ha tenido una trascendencia e implicación tan fuerte en el arbitraje de inversiones, que podemos considerar que en el futuro constituirá parte inseparable de la realidad moderna del arbitraje internacional ${ }^{91}$.

Ante el vacío existente en las reglas institucionales comentadas con anterioridad, y establecida la necesidad de regular dicha actividad $^{92}$, resulta de gran interés comentar los avances recientes que han tenido algunas legislaciones al respecto. En este sentido, las Reglas de Arbitraje de Inversión de la Comisión Internacional de Arbitraje Económico y Comercial de China de $2017^{93}$, establecen como obligatorio que la parte que recibe el financiamiento debe notificar al resto de las partes del litigio, al tribunal y a la propia institución arbitral,

HARWOOD, M.K., BATIFORT, S.N., TRAHANAS, CH., Third-Party Funding..op.cit... nota 86, p 98.

90 Tal y como lo afirma CATHERINE al expresar, que la reciente y extensa expansión de la financiación de terceros es "parte integrante de una desregulación más global y sistemática de la profesión legal"; ROGERS, C.A., "Gamblers, Loan Sharks \& Third-Party Funders”, Penn State Law Research Paper, No. 51, 2013, p. 3-7. Disponible en: https://ssrn.com/abstract=2345962, consultado el 14/06/2020; Véase también, CHAN, E.: "Funding International Arbitration", New Zealand Law Journal, 2014, p. 45.

91 KALICKI, J.E., "Third-Party Funding in Arbitration: Innovations and Limits in Self-Regulation", Kluwer. Arb, 2012, Part 2 of 2. Disponible en: http://kluwerarbitrationblog.com/blog/2012/03/14/thirdparty-funding-in-arbitration-innovationsand-limits-in-self-regulation-part-2-of-2, consultado el 15/07/2019. En el caso Giovanni Alemanni and others vs. Argentine Republic, Case ICSID No. ARB/07/8, 2015, en el cual, el tribunal arbitral expresó: "the practice is by now so well established both within many national jurisdictions and within international investment arbitration that it offers no grounds in itself for objection". Véase también, BOGART, C.P.: "Third Party Funding in Action: Alemanni v Argentina", 2015. Disponible en: http:// www.burfordcapital.com, consultado el 15/07/2019; BOOLIERIS, E., Third-Party Funding: the effect of the growing third-party funding industry in international arbitration on New Zealan, Victoria University of Wellington, 2015, p. 25; SEVILA, E., ANDRÉS O., La financiación por terceros en el arbitraje internacional...op.cit...nota 37, p. 11; informe ICCA-Queen Mary Task Force on Third-Party Funding In International Arbitration....op.cit..., p. 5.

92 HODGES, CH., PEYSNER, J., NURSE A., "Litigation Funding: Status and Issues", Oxford Legal Studies Research Paper, No. 55, 2012, p. 151; EBERHARDT, P., OLIVET, C., "Profiting from injustice. How law firms, arbitrators and financiers are fuelling an investment arbitration boom", Corporate Europe Observatory, 2012, p.58, STEINITZ, M., "Whose claim is it anyway? Third-party Litigation Funding", Transnational Dispute Management, Vol. 8, No. 4, 2011, p. 1278.

93 Conocida por sus siglas en inglés como CIETAC - China International Economic and Trade Arbitration Commission. 
la naturaleza del acuerdo de financiación, así como la identidad y dirección del tercero, resultando lo más relevante en estas reglas la facultad que se le otorga al propio tribunal arbitral para poder exigir a la parte financiada información relevante de dicho acuerdo arbitral ${ }^{94}$. En este mismo sentido, el Acuerdo de Libre Comercio entre la Unión Europea y Vietnam de $2018^{95}$, también establece un deber amplio de revelación del tercero financiador, incluyendo el acuerdo de financiación.

Cabe destacar el papel que ha tenido el Parlamento de Singapur ${ }^{96}$ con la aprobación de la Civil Law (Amendment) Bill - Third Party Funding for Arbitration and Related Proceedings. También, el Acuerdo Económico y Comercial Global entre Canadá y la Unión Europea (CETA-por sus siglas en inglés) ${ }^{97}$ y el Tratado Trasatlántico de Libre Comercio e Inversión (TTIP), los cuales establecen la obligatoriedad de revelación del tercero financiador. Pero este deber de revelación es limitado, refiriéndose únicamente al nombre y dirección del financiador. En este mismo sentido, el Consejo de la International Bar Association (IBA) introduce ciertas modificaciones en sus directrices sobre el conflicto de interés en el arbitraje internacional, con el objetivo de actualizarlas y adecuarlas a la actividad propia de la financiación por terceros. En efecto, la Directriz 6.b dispone que "si una de las partes fuere una persona jurídica, cualquier persona jurídica o física que tenga una relación de control sobre dicha persona jurídica, o que tenga un interés económico directo en, o deba

\footnotetext{
94 Art. 27, de las Reglas de Arbitraje de CIETAC, 2017.

95 FERNÁNDEZ MASIÁ, E., La financiación por terceros en el arbitraje internacional...op.cit..nota 51, p. 216.

96 En enero de 2017, el Parlamento de Singapur aprueba la Civil Law (Amendment) Bill - Third Party Funding for Arbitration and Related Proceedings. Con anterioridad a dicha ley, era prohibida la financiación por terceros, lo que demuestra, según reconocen ANDRÉS ROS y SEVILA, "el posicionamiento de Singapur en la solución de controversias internacionales"; ANDRÉS ROS, O., SEVILA, E., La financiación por terceros en el arbitraje internacional,...op.cit...nota 85, pp. 2, 11. En consonancia, también, con lo establecido en el Proyecto de Reglas de Arbitraje del Centro Internacional de Singapur, Art. 23.1, las cuales establecen el deber de revelación amplio del TPF. Disponible en: http://www.siac.org.sg/images/stories/articles/ rules/IA\%20Rules\%20\%28rev\%2020160115\%29.pdf, consultado el 23/05/2020.

97 Véase, CETA, Capítulo 8, Sección A, Art. 8.26. Financiación de una tercera parte, el cual establece: 1. Cuando exista financiación de una tercera parte, la parte en la diferencia que se beneficie de ella deberá comunicar a la otra parte en la diferencia y al tribunal el nombre y la dirección del financiador de la tercera parte. 2. La comunicación se efectuará en el momento de la presentación de la demanda, o, en caso de que el acuerdo de financiación haya concluido o de que la donación o la subvención se hayan realizado tras la presentación de una demanda, sin dilación alguna en cuanto concluya el acuerdo o se realice la donación ola subvención. Disponible en: https://www. boe.es/doue/2017/011/L00001-01079.pdf, consultado 23/05/2020.
} 
indemnizar a una parte por, el laudo que se vaya a emitir en el arbitraje podrá considerarse que ostenta la identidad de dicha parte". En este sentido, las Directrices de la IBA intentan equiparar al tercero financiador con la parte financiada, obligando a esta última a revelar cualquier relación existente, teniendo en cuenta la propia nota explicativa a esta disposición cuando refiere que "los terceros financiadores y las aseguradoras con relación a la disputa pueden tener un interés económico directo en el laudo, y por tanto pueden considerarse como equivalentes a la parte. A estos efectos, los términos 'tercero financiador' y 'aseguradora' se refieren a cualquier persona o entidad que contribuya con fondos, $u$ otro tipo de apoyo material, al desarrollo del proceso en interés de la demanda o defensa del caso y que tenga un interés económico directo en, o un deber de indemnizar a una parte por, el laudo que se vaya a emitir en el arbitraje" ${ }^{98}$.

En relación con lo anterior, la Directriz 7 a) establece que: "Cada parte deberá informar al árbitro, al Tribunal Arbitral, a las demás partes y a la institución arbitral o a cualquier otra institución nominadora (si la hubiere) sobre cualquier relación directa o indirecta que hubiere entre el árbitro y la parte (o cualquier otra sociedad del mismo grupo de sociedades o un individuo con una relación de control sobre la parte en el arbitraje), o entre el árbitro y cualquier persona o entidad con un interés económico directo en, o un deber de indemnizar a una parte por, el laudo que se emita en el arbitraje. Cada parte informará a iniciativa propia lo antes posible". La obligación de revelación ahora presente en las Directrices, a diferencia de la versión anterior, tienen un alcance mayor. La presente norma abarca también a aquellos terceros (personas física o jurídica) con un interés económico en el laudo a dictarse en el arbitraje, incluyendo el tercero financiador. Creemos que el objetivo final de la presente norma no es otro que preservar la integridad del procedimiento arbitral.

Sin embargo, con excepción de las Reglas de China International Economic and Trade Arbitration Commission (Comisión Internacional de Arbitraje Económico y Comercial de China CIETAC) y el Acuerdo de Libre Comercio entre la Unión Europea y Vietnam, el resto de las normas comentadas con anterioridad son muy escuetas al respecto, únicamente establecen la obligatoriedad de notificación, por la parte financiada, de la información referente al nombre y domicilio del tercero financiador. Creemos, que dicha información no resulta del todo significativa o relevante, y que constituye

98 FERNÁNDEZ MASIÁ, E., La financiación por terceros en el arbitraje internacional...op.cit..nota 51, p. 210. 
una obligación de publicación parcial de la información del tercero financiador. Esto impide lograr una mayor transparencia en la actividad de financiación por terceros en el arbitraje internacional.

Algunos estudiosos del tema ${ }^{99}$ consideran que el acuerdo de financiación es una cuestión privada entre la parte financiada y el tercero financiador, un asunto entre ellos, que no debe ser revelado, salvo voluntad de las propias partes del acuerdo ${ }^{100}$. Consideran también que no debe extender sus efectos a terceros. Esto justificaría que los árbitros, en principio, no fuesen competentes para adentrarse en cuestiones de fondo del acuerdo de financiación. Que los árbitros solo se deben a la disputa entre las partes para la cual fueron nombrados, siendo el acuerdo de financiación independiente al procedimiento arbitral ${ }^{101}$.

Sin embargo, creemos que dichos motivos no deben ser un impedimento para que las partes involucradas en el litigio puedan conocer el acuerdo de financiación. El acuerdo de financiación tiene una influencia directa en la eficacia del procedimiento arbitral. De manera que cualquier problema que surja entre la parte financiada y el tercero financiador repercutirá directamente en el propio proceso arbitral ${ }^{102}$. Es lo que ocurrió en el caso $S \& T$ Oil

99 Gran parte de los estudiosos en el tema argumentan que si el tribunal arbitral no tiene conocimiento de la presencia de un tercero en el procedimiento, la presencia del tercero financiador no tendrá ninguna relevancia o efecto en la decisión final. Otros alegan que la no revelación del tercero financiador en el procedimiento, evitaría la existencia de posibles litigios auxiliares que pueda provocar la otra parte, con el objetivo de distraer la atención de los árbitros respecto de la disputa principal. Véase, BENCH NIEUWVELD, L., SHANNON, V., Third-Party Funding in International Arbitration...op.cit..nota 54, p. 26.

100 Tal y como sucedió en el caso Oxus Gold plc vs. Republic of Uzbekistan, the State Committee of Uzbekistan for Geology \& Mineral Resources, and Navoi Mining \& Metallurgical Kombinat, UNCITRAL, 2012, en el cual, la parte Demandante (Oxus) celebra un acuerdo de financiación con Calunius Capital LLP, para cubrir los gastos de la disputa. Y, por voluntad de la propia parte financiada, se revela en el proceso arbitral la presencia del tercero financiador.

101 DE BRABANDERE, E., LEPELTAK, J., Third Party Funding in International Investment Arbitration,...op.cit...nota 38, p. 4. En este sentido, existen pronunciamientos de quienes, reconociendo que los árbitros en una cuestión inicial no tienen jurisdicción sobre terceros financiadores, defienden la existencia de cuestiones tales como: las relacionadas con el conflicto de intereses; seguridad y adjudicación de costos y; la divulgación de la presencia del tercero financiador, como elementos que pueden contribuir a la existencia de un proceso arbitral más justo. ROGERS, C.A., Gamblers, Loan Sharks \& Third-Party Funders...op.cit..nota 88, p. 45.

${ }_{102}$ DE MORPURGO, M., "A Comparative Legal and Economic Approach to Third-Party Litigation Funding", Cardozo Journal of International and Comparative Law, Vol. 19, 2011, p. 352. 
Equipment \& Machinery Ltd. vs. Rumania ${ }^{103}$, en relación con el caso S\&T Oil Equipment \& Machinery vs. Juridica Investment Limited $^{104}$. La disputa se resume en las reclamaciones derivadas de desacuerdos contractuales entre las partes y la rescisión por parte del Gobierno rumano de ciertos acuerdos de privatización celebrados con el inversor, S\&T Oil Equipment \& Machinery Ltd., seguidas de la cancelación de las acciones de la empresa, de sus activos rumanos y la re-privatización de los activos. Cabe señalar que, en el presente caso, el deterioro de la relación entre el demandante parte financiada- y el tercero financiador generó incumplimientos de pago, lo que produjo grandes tensiones entre las partes que no llegaron a buen término, y que trajo como consecuencia la interrupción directa del procedimiento arbitral ante el CIADI.

Por todo lo anterior, creemos que las instituciones arbitrales deben actualizar, sus reglas. Establecer en ellas, no solo la obligación de divulgación del nombre y domicilio del tercero financiador y la simple existencia del acuerdo o contrato de financiación, información que consideramos muy concisa, sino también deben exigir, ante un ISDS, la obligación de divulgación de las condiciones del acuerdo de financiación. Esta divulgación puede resultar útil, tanto para el tribunal como para el resto de las partes en litigio ${ }^{105}$ — cuestión que no deja de ser controvertida en el arbitraje internacional-, en busca de una mayor transparencia en la resolución del caso. Consideramos que el solo conocimiento de la existencia de un acuerdo de financiación, o algunas de sus condiciones, no resultan suficiente para un análisis detallado y general del mismo. Creemos que el análisis de un contrato, como resulta ser el acuerdo de financiación por terceros, no puede darse aisladamente, analizando algunas de sus condiciones,

103 S\&T Oil Equipment \& Machinery Ltd. vs. Romania, ICSID Case No. ARB/07/13, 2007. Order of Discontinuance of the Proceeding, 2010. Disponible en: https://www.italaw.com/sites/default/files/case-documents/ita0743.pdf, consultado el 23/05/2020. Para mayor conocimiento al respecto, véase, CREMADES Jr, B.M.: Third Party Litigation Funding...op.cit.., pp. 25-32.

104 Véase, U.S. District Court, S.D. Texas, Houston Division, $S \& T$ Oil Equipment \& Machinery vs. Juridica Investment Limited, Civil Action No. H-11-0542, Memorandum and Order, 2011. Cuyo Memorandum se encuentra disponible en: https://docs.justia.com/cases/federal/district-courts/texas/ txsdce/4:2011cv00542/865197/29, consultado el 23/05/2020.

105 Ante la ausencia de consenso a nivel internacional respecto a la publicación o no, de todo el acuerdo de financiación o parte de este. Somos partícipe de la importancia que tiene el conocimiento, al menos, de aquellos aspectos del acuerdo de financiación que tenga relevancia para el procedimiento arbitral. En este sentido, la comunidad arbitral internacional puede tener como referencias, la experiencia a nivel nacional de algunos países, como pueden ser Australia y Nueva Zelanda. Véase, al respecto, BOOLIERIS, E., Third-Party Funding...op.cit...nota 89, pp. 37-38. 
sino que debe hacerse en su totalidad, el contrato en general, lo que permitirá una mejor interpretación de este y un mejor conocimiento de la posible influencia que puede tener en el procedimiento arbitral.

Existen condiciones del acuerdo de financiación cuya revelación o conocimiento, por el tribunal y las partes, pueden resultar muy significativas para el procedimiento arbitral, como pueden ser las obligaciones y derechos de las partes; la obligación de verificaciones de conflictos de intereses en relación con todas las partes del proceso arbitral antes de acordar el financiamiento; las fuentes de financiación o posición financiera ${ }^{106}$, teniendo en cuenta la influencia que tiene el tercero financiador en el procedimiento arbitral y si se ha pactado la responsabilidad del financiador ante los costes adversos generados durante el procedimiento, de ser así, hasta qué punto o en qué medida resulta posible. Por ejemplo, en caso de que la parte financiada —el demandante-, no resulte vencedora en el procedimiento y la parte demandada le exija pagar los costes en los que ha incurrido, lógicamente, el demandante no tendrá cómo hacer frente a dichos costes, teniendo en cuenta que el mismo ha tenido que acudir a un tercero financiador para poder emprender el procedimiento arbitral. Esto genera, no solo la imposibilidad del tribunal arbitral de poder adjudicarle los costes al tercero financiador ${ }^{107}$, sino

106 Existen algunas empresas que actúan como terceros financiadores en un proceso arbitral que, por su condición de empresas públicas con capital social negociado, deben divulgar públicamente su posición financiera, por ejemplo: IMF Australia Ltd; Burford Capital Limited y Juridica Investments Limited. Sin embargo, existen otras que no están obligadas a hacerlo por la condición de empresa privada o fondos privados de inversión, como son: Woodsford Litigation Funding Limited; Harbour Litigation Funding Limited; Calunius Capital LLP y, Vannin Capital. Para cualquier tercero financiador, sin tener en cuenta su tipicidad, lo más importante es que ven el riesgo que son capaces de asumir como un atractivo porque el mismo no está correlacionado con el mercado, en este sentido, MOLOT, ha expresado, "Hedge funds, and some traders at investment banks, have long viewed litigation risk as attractive because it is uncorrelated with markets-the outcome of a lawsuit will not depend upon fluctuations in the stock or bond markets. For this reason, hedge funds seeking a diverse portfolio of investments have tried to earn returns by betting on litigation", MOLOT, J.T.: "Litigation Finance: A Market Solution to a Procedural Problem", Georgetown Law Journal, Vol. 99, 2010, p. 65-115. Puede verse también, una lista más exhaustiva sobre terceros financiadores en: GARBER, S., "Alternative Litigation Financing in the United States", Occasional Paper, 2010, pp. 11,14-15. Disponible en: https://www.rand.org/content/dam/rand/pubs/occasional_papers/2010/ RAND_OP306.pdf, así como, en la siguiente lista de financiadores y corredores. Disponible en: http://www.international-arbitration-attorney.com/third-party-funders, ambos sitios web fueron consulatdos el 23/05/2020.

107 RUBINS, N., "In God We Trust, All Others Pay Cash: Security for Costs in International Commercial Arbitration", American Review International Arbitration, Vol. 11, 2000, p 361. 
también el riesgo de que el demandado no pueda recuperar los gastos en los que ha incurrido ante un demandante insolvente.

El reconocimiento en las reglas arbitrales de la obligatoriedad de revelación del tercero financiador y las particularidades del acuerdo arbitral a todas las partes involucradas en el caso ${ }^{108}$, allanaría el camino de evitar posibles contradicciones con el requisito de confidencialidad existente en varias jurisdicciones respecto a la relación de confidencialidad abogado-cliente ${ }^{109}$, en relación con la información de la parte contendiente que le es exigida por el tercero financiador, bien al abogado, representante legal de la parte a financiar, o directamente a la propia parte que recibe el financiamiento, para ser estudiada y analizada por el financiador antes de tomar la decisión de financiar el caso, así como con el acuerdo de confidencialidad que suele existir en el propio acuerdo de financiación existente entre la parte financiada y el tercero financiador ${ }^{110}$. Por lo que existe el riesgo de que dicha confidencialidad, el deber abogado-cliente, se vulnere una vez entregada la documentación al tercero financiador ${ }^{111}$.

108 En este sentido, se pronuncia también TRUSZ, alegando que las instituciones arbitrales deben de enmendar sus reglas para imponer una obligación de divulgación del tercero financiador, pero teniendo en cuenta dos condiciones fundamentales: primero, que "el requisito de divulgación se aplica a todas las partes involucradas, ya sea que opere a través de una aseguradora o el propio abogado" y, segundo "la institución arbitral garantice que esto no afectará el procedimiento de arbitraje”; TRUSZ, J.A., Full Disclosure...op.cit...nota 38, p. 1676.

109 Algunos autores consideran que "la existencia del acuerdo de financiación puede representar una amenaza para el privilegio abogado-cliente y el deber de confidencialidad entre ambos. KHOURI, S., HURFORD, K., Third party funding for international arbitration...op cit...nota 50, p. 12, FRISCHKNECHT, A., SCHMIDT, V., "Privilege and Confidentiality in Third Party Funder Due Diligence: The Positions in the United States and Switzerland and the Resulting Expectations Gap in International Arbitration", Transnational Dispute Management, Vol. 8, No.4, 2011, pp. 21-22. Debe tenerse presente que "algunas jurisdicciones reconocen una excepción de interés común para le privilegio abogado-cliente. Sin embargo, dicha excepción no es uniforme para todas las jurisdicciones a nivel internacional, de ahí el carácter incierto en su aplicación a las comunicaciones confidenciales"; ROGERS, C.A., Gamblers, Loan Sharks \& Third-Party Funders.... op.cit...nota 88, p. 26; MERIAM, N., ALRASHID, J. W., LAIRD, J., "Impact of Third Party Funding on Privilege in Litigation and International Arbitration", Dispute Resolution International Law, Vol. 6, 2012, p. 108; LEGG M., "Litigation Funding in Australia: Identifying and Addressing Conflicts of Interest for Lawyers", Chamber Institute for Legal Reform, 2012, p. 20.

110 ROSS, A., "The Dynamics of Third-Party Funding", Global Arbitration Review, Vol. 7, No. 1, 2012, p. 19; ATTRILL, W., "Ethical issues in litigation funding", IMF (Australia), 2009, p.8.

111 ADELINE, A., PERRIN, L., "Third-party funding of arbitration in France", Squire Sanders International Arbitration News, 2013, Disponible en: www.squire-

(C) UNED. Revista de Derecho UNED, núm. 28, 2021 
Cuestiones estas que facilitarían que las disposiciones contractuales en materia de confidencialidad no impidan que la parte en litigio que no resulta financiada - generalmente el demandadopueda obtener acceso a la información en posesión del financiador si son relevantes para el asunto en cuestión. Podría suceder, incluso, que dicha información se encuentre protegida bajo el principio de confidencialidad. Cabría entonces preguntarnos, ¿hasta qué punto el tercero financiador estaría obligado a mantener o preservar como confidencial dicha información? En muchos ordenamientos jurídicos se establece el deber de confidencialidad abogado-cliente como un principio esencial de la relación, para evitar la revelación de información y la existencia de conflicto de interés. Sin embargo, no existe a nivel internacional una obligación o un deber de protección de la información o confidencialidad por los terceros financiadores, de modo que los propios acuerdos de financiación en el arbitraje internacional tienen el privilegio de disfrutar de mucho menos control que los acuerdos de financiación de litigios respecto de los ordenamientos nacionales que lo regulan ${ }^{112}$. En consecuencia, consideramos que, ante un arbitraje de inversiones, resulta necesaria la divulgación total del acuerdo de financiación, no así, ante un arbitraje comercial internacional - en ausencia de un Estado o institución pública-. En éste, consideramos que dicha revelación puede ser parcial, no total, teniendo en cuenta la privacidad y confidencialidad existente.

A nuestro juicio, la financiación por terceros en el arbitraje internacional es el vehículo innovador más acorde para lograr la solución de diferencias de aquellos que, siendo partes en la controversia, no cuenten con los recursos necesarios suficientes dado los altos costes del proceso. No es menos cierto que, en determinadas

sanders.com/files/Publication/bfa63ce4-9bcd-44fc-8eae-08f219a66927/Presentation/ PublicationAttachment/abdbf613-9b29-4060-b2d5-0975f2ab4dbd/International-Arbitration-News-March-2013.PDF, consultado el 25/06/2020; BERTRAND, E., "The Brave New World of Arbitration: Third-Party Funding", ASA Bulletin, Vol. 3, 2011, pp. 607-615, teniendo en cuenta que el privilegio de interés común es considerado como una excepción al privilegio abogado cliente, el cual protege las comunicaciones cuando dos o más clientes consultan simultáneamente con un abogado en asuntos de interés común. En la actualidad, algunos autores dejan abierta la posibilidad de si resulta posible que las comunicaciones existenten con el tercero financiador pueden incluirse en el grupo de personas a quienes se les aplica el interés común. Hasta la fecha de realización del presente trabajo, no se ha dado caso alguno en el que se haya producido tal situación. Véase, ALRASHID, J. W., LAIRD, J., Impact of Third Party Funding on Privilege...op.cit...nota 107, p. 108; NIEUWVELD, L., SHANNON, V., Third-Party Funding in International Arbitration,...op.cit..., pp. 55-56.

112 ROGERS, C.A.: Gamblers, Loan Sharks \& Third-Party Funders...op.cit... nota 54, p. 44. 
jurisdicciones, el poder litigar se ha convertido prohibitivamente costoso, o, como comentamos antes, teniendo dichos recursos económicos, no asumen el riesgo de perderlos, prefiriendo solucionar el mismo a través de una institución totalmente privada como es el arbitraje con el apoyo financiero de un tercero ${ }^{113}$, asumiendo los riesgos inherentes a este tipo de $\operatorname{actividad}^{114} \mathrm{y}$, de esta manera, poder tener acceso a la justicia ${ }^{115}$.

La financiación por terceros no solo tiene como cuestión principal la financiación de los honorarios del procedimiento arbitral de la parte a financiar, sino también el financiamiento de su posible responsabilidad en un caso dado. De esta forma, ha motivado que los propios financiadores puedan ofrecer un amplio paquete de financiamiento en el cual incluyen, además, un seguro; o sea, la existencia de posibles aseguradoras, siendo una tendencia en el arbitraje de inversiones. En este sentido, resultan beneficiadas de las políticas nacionales de los Estados o las medidas de expropiación, como causas de origen del procedimiento arbitral, los grandes financiadores o grupos privados ${ }^{116}$.

Resulta innegable que, sin olvidar el carácter privado que entraña el arbitraje internacional en su función de resolución de litigios, también desempeña una función pública en auxilio o sustitución, según sea el caso, de los órganos judiciales. No se puede continuar percibiendo el arbitraje internacional como una amenaza potencial del sistema judicial, mucho menos negar la complementariedad de este para con los órganos judiciales. Cuestiones

113 Para algunos ordenamientos jurídicos, compatibles con la financiación por terceros en el arbitraje internacional. La financiación por terceros resulta la primera opción en caso de imposibilidad financiera para participar en el arbitraje, tal y como sucede en el Derecho brasileño. Véase, CASADO FILHO, N., "O Financiamento de Arbitragens por terceiros”, Direito Internacional em Análise, Vol. 4, 2015, p. 20.

114 RANDAZZO, S., "Third Party Funding of Lawsuits Gains Ground But Raises Eyebrows", Daily J., 2010; RODAK, M., "It's about Time: A System Thinking Analysis of the Litigation Finance Industry and Its Effect on Settlement", University of Pennsylvania Law Review, 2006, p. 517; AFFAKI, G., "A financing is a financing is a financing", en CREMADES, B., DIMOLITSA, A. (eds.): Dossier X: Third-party Funding in International Arbitration, ICC Publishing S.A., Paris, 2013, p. 11.

115 DE MORPURGO, M., A Comparative Legal and Economic...op.cit..nota 100, pp. 381-382; KANTOR, M., “Third-Party Funding in International Arbitration: An Essay About New Developments", ICSID Rev., Vol. 24, No. 1, 2009, p. 73.

116 También se han ido generando otras formas de financiación que, si bien no han sido muy generalizadas en la práctica, pueden tener un mayor desarrollo y utilización en el futuro, por ejemplo: la emisión de valores en mercados bursátiles, tal y como ha realizado la compañía canadiense Crystallex en el arbitraje frente a Venezuela ante el CIADI; Véase, FLÉCHET C., Summary report of the first session held...op.cit...nota 46, p. 211. 
que siguen siendo muy habituales por quienes no son buenos receptores del sistema arbitral.

Somos conscientes del rol trascendental que deben desarrollar las instituciones arbitrales en relación con la financiación por terceros. Queda demostrado que la financiación por terceros, hoy en día, es una opción indiscutible para muchos demandantes en el arbitraje internacional. Es por ello, que se hace necesario mayor información, más conocimiento de la práctica de la financiación por terceros, de sus efectos, lo que permitirá comprender su naturaleza ${ }^{117} \mathrm{y}$, de esta manera, facilitar una regulación de dicha actividad en el ámbito del arbitraje internacional. Se hace necesario, a nivel internacional, un pronunciamiento de las principales instituciones jurídicas que favorezca la regulación internacional de la financiación por terceros. Que permita que las propias instituciones arbitrales puedan modificar o revisar sus reglas para regular la financiación por terceros sin que colisionen con los ordenamientos jurídicos nacionales. De lo contrario, las instituciones arbitrales seguirán actuando reticentemente para actualizar sus reglas.

La falta de regulación puede también constituir un impedimento real para el desarrollo de la financiación por terceros, pudiendo provocar un desaliento de las partes a la hora de solicitar el apoyo financiero de un tercero, teniendo en cuenta que sus derechos y obligaciones no se encuentran protegidos por normas o reglas vinculantes. Debe tenerse siempre presente que el tercero financiador ha llegado al proceso para obtener ganancias, es el único de todos los presentes cuya presencia es únicamente lucrativa, cuyo objetivo meramente distinto puede traer innumerables problemas. Cuestión que algunos autores han solido llamar "el triángulo de Bermudas de intereses divergentes"118, y que constituyen, sin lugar a duda, un gran desafío para la comunidad arbitral internacional. Mientras tanto, tendrán que continuar los tribunales arbitrales haciendo uso de sus recursos — bastante escasos- para conservar y proteger el procedimiento arbitral y las partes de posibles iniquidades del tercero financiador ${ }^{119}$.

117 ROGERS, C.A., Gamblers, Loan Sharks \& Third-Party Funders....op.cit... nota 88, p. 44; ROGERS, C.A.: "The Vocation of International Arbitrators", American University International Law Review, Vol. 20, No. 5, 2005, pp. 959-960.

118 LAMM, C., HELLBECK, E.R., "Third-party funding in investor-state arbitration", en CREMADES, B.M., DIMOLITSA, A. (eds.), Dossier X: Third-party Funding in International Arbitration, ICC Publishing S.A., Paris, 2013, p. 107.

119 KALDERMIS, D., GIBBS, P., "Third-party funding in international arbitration - lessons from litigation?”, Kluwer Arbitration, 2014, p. 2. Disponible en: http:// kluwerarbitrationblog.com, consultado el 24/06/2020. 


\section{LOS EFECTOS DE LA FINANCIACIÓN POR TERCEROS EN EL ARBITRAJE INTERNACIONAL}

La prevalencia de la financiación por terceros en el arbitraje internacional ha tenido un impacto directo en las estrategias a seguir en el desarrollo de los procedimientos arbitrales con presencia de terceros financiadores en el ámbito internacional. Ningún Estado está exento de afrontar los efectos de dicha actividad, debido a la proliferación del arbitraje internacional, especialmente, del arbitraje de inversiones, teniendo en cuenta el aumento de los tratados, ya sean bilaterales o multilaterales que lo regulan.

El crecimiento de la actividad de financiación por terceros puede tener un efecto directo en los propios Estados, no solo para incentivar la regulación de dicha actividad a nivel nacional, según las características de los ordenamientos jurídicos que así lo permitan ${ }^{120}$, sino también, para que los Estados puedan hacer mayor presión a la comunidad jurídica internacional en la búsqueda de un consenso y el logro de una regulación internacional sobre la financiación por terceros en el arbitraje internacional.

Este tipo de actividad en el arbitraje internacional, sin la correspondiente divulgación, puede influir en los costos del procedimiento ${ }^{121}$ y en la duración de estos, e incluso, podría incidir, en determinadas ocasiones, en la existencia de comportamientos que pueden generar una "violación de la buena fe procesal de las partes en el procedimiento"122. Además, introducir distorsiones en la relación abogado-cliente ${ }^{123}$ entre la parte a financiar y su abogado y en

120 BOOLIERIS, E., Third-Party Funding...op.cit...nota 89, p. 56.

121 Mucho se ha escrito respecto a la influencia de terceros financiadores en los costes del procedimiento arbitral, para un mejor análisis del tema puede consultarse, entre otros: KALICKI, J., "Security for Costs in International Arbitration", Transnational Dispute Management, No. 5, 2006. Disponible en: www.transnationaldispute-management.com/article.asp?key=827, consultado el 14/09/2020; KIRTLEY, W., WIETRZYKOWSKI, K., "Should an Arbitral Tribunal Order Security for Costs When an Impecunious Claimant Is Relying upon Third-Party Funding?", J. Int. Arb, Vol. 30, No. 1, 2013, pp. 26-27; LAMM, C., HELLBECK, E.R., Third-party funding....op.cit.,nota 116, p. 111.

${ }_{122}$ CREMADES, B.M., Third party funding in international arbitration.... op.cit., nota 37, p. 7.

123 Estos efectos de la financiación por terceros constituyen hoy grandes preocupaciones respecto a la actividad, tanto es así, que hasta la propia Cámara de Comercio de los Estados Unidos ha manifestado su preocupación respecto a la influencia del tercero financiador en la relación abogado-cliente de la Parte financiada. Véase, LOWE R., "Investment arbitration claims could be traded like derivatives", IBA Global Insigh, 2013. Disponible en: http://www.ibanet.org/Article/Detail. aspx?ArticleUid=02decc8d-bf67-4b86-a023-f2ef2aa4843b, consultado el 14/09/2020.

(C) UNED. Revista de Derecho UNED, núm. 28, 2021 
la naturaleza confidencial del arbitraje. Todo ello podría tener como principal causa la ausencia de normas procesales internacionales.

Básicamente, si nos encontramos ante un arbitraje comercial internacional, en el cual, el solo hecho de que una de las partes le presente el caso a un tercero financiador para que evalúe la posibilidad de financiarlo, puede poner en peligro la confidencialidad ${ }^{124}$. El tercero financiador no solo valorará la probabilidad de éxito a obtener, sino que, para poder llevar a cabo un minucioso estudio del caso, necesitará también que la parte a financiar le proporcione información respecto al procedimiento arbitral, como puede ser el acuerdo de arbitraje, la composición del tribunal arbitral, las leyes aplicables al arbitraje, la jurisdicción donde se ejecutará el laudo y la duración probable del arbitraje ${ }^{125}$. Además, si resulta viable la financiación, y el tercero financiador así lo decide, el mismo solicitará al reclamante - parte financiada- que le mantenga informado del procedimiento, o sea, existirá una segunda fase en la cual el tercero financiador llevará un monitoreo constante del procedimiento arbitral.

El tercero financiador no está sujeto al acuerdo arbitral entre las partes en litigio y mucho menos a la confidencialidad ${ }^{126}$. Pero, al conocer las particularidades del caso mediante la información que le proporcione la parte financiada, ésta última puede correr el riesgo de incumplir sus obligaciones, llegando a aportar información que podría ocasionar consecuencias nefastas para la otra parte - no financiada- en litigio. Información de la que se sustentará el financiador y que podría, en futuros litigios, hacer valer contra la propia parte no financiada.

En relación con los costes del procedimiento, lo más relevante en cuanto a los efectos del tercero financiador es lo concerniente a los términos del acuerdo de financiación que les permita una mayor seguridad a las partes, principalmente a la demandada, en relación con los costes del procedimiento. Es decir, si en el acuerdo de financiación se ha establecido alguna obligación, cautio judicatum solvi (security for costs), que pueda hacer responsable al financiador de los costes adversos contra el reclamante. Y, en caso de que el financiador decida rescindir el acuerdo de financiación, en qué

124 VON GOELER, J., Third-Party Funding in International Arbitration and Its Impact on Procedure, Kluwer Law International, The Netherlands, Vol. 35, 2016, p. 298.

125 DOS SANTOS, C., Third-party funding in international comercial arbitration...op.cit., nota 53, p. 922.

${ }_{126}$ VON GOELER, J., Third-Party Funding in International Arbitration... op.cit., nota 122, p. 299. 
condiciones lo hará ${ }^{127}$. Un procedimiento arbitral iniciado por un demandante financiado conlleva el riesgo de ocasionarle al demandado importantes gastos asociados al litigio, sin que el demandante cuente con la capacidad financiera necesaria para poder enfrentarlos. Además, pueden surgir ciertas circunstancias que pueden hacer más difícil la situación del demandado, como pueden ser: i) que la demanda sea rechazada por el tribunal; ii) que el tercero financiador decida retirar el financiamiento provocando la interrupción del procedimiento; iii) que el procedimiento continúe su curso y el demandado obtenga una resolución favorable, es decir, se condene en costa al demandante financiado. Encontrándonos ante tales situaciones, surge entonces la preocupación de cómo garantizar los derechos del demandado de recuperar los gastos generados durante el procedimiento.

La caución o cautio judicatum solvi, como medida provisional, permite "preservar los activos por los que se puede cumplir un laudo posterior" 128 , siendo una de las opciones con las que cuenta la parte demandada en un momento del proceso. Es una figura jurídica muy utilizada en el ámbito anglosajón que, con el transcurso del tiempo, se ha ido incorporando al arbitraje internacional. Como medida cautelar, permite que la parte demandada pueda solicitar al tribunal arbitral que ordene al demandante constituir una caución, es decir, que pague en concepto de fianza ${ }^{129}$ una cierta cantidad de dinero con el objetivo de garantizar que la parte demandada pueda recuperar las costas del arbitraje si el procedimiento arbitral es resuelto mediante un laudo que condene en costas al demandante ${ }^{130}$. En la

127 En la actualidad, el tercero financiador puede rescindir el contrato de financiación dejando al Demandado sin posibilidades reales de poder recuperar los costes en los que ha incurrido, sin recurso alguno que permita la recuperación de los mismos, tal y como ha ocurrido en varios casos de arbitraje de inversiones. Véase, TREVINO, C., "One of Three ICSID Argentine Bond Arbitrations Collapses Due to Lack of Funding", Investment Arbitration Reporter, 2015; HARWOOD, M.K., BATIFORT, S.N., TRAHANAS, CH., Third-Party Funding..op.cit., nota 86., p. 115.

128 Las propias reglas de la CNUDMI, reconocen claramente la facultad del tribunal arbitral de poder establecer medidas provisionales; PETROCHILOS, G., "Interim Measures under the Revised UNCITRAL Arbitration Rules", ASA Bulletin, Vol. 28, No. 4, 2010, p. 885. En este mismo sentido, véase también, WIRTH, M., "Interim or Preventive Measures in Support of International Arbitraion in Switzerland", ASA Bulletin, Vol. 18, No. 1, 2000, pp. 32-40; YESILIRMAK, A., Provisional Measures in International Commercial, Arbitration. Kluwer Law International, London, 2005, p. 48.

129 FERNÁNDEZ MASIÁ, E., La financiación por terceros en el arbitraje internacional...op.cit., nota 51, pp. 216-217.

130 KIRTLEY, W., WIETRZYKOWSKI, K., Should an Arbitral Tribunal Order Security for Costs...op.cit., nota 119, pp. 19-21. 
actualidad, ha sido objeto de interesantes debates por la comunidad arbitral si entre las facultades concedidas al tribunal arbitral este puede decretar una caución al inversor financiado con el fin de garantizar el pago de una condena en costas en su contra o de los gastos adversos generados durante el procedimiento.

Sin negar las amplias facultades con las que cuenta el tribunal, incluyendo la de ordenar al demandante que preste una caución, la doctrina no ha sido unánime al respecto, existiendo dos corrientes de pensamiento. Por un lado, nos encontramos algunos autores que consideran que es perfectamente posible ${ }^{131}$, al considerarlo como una facultad del tribunal arbitral para decretar medidas cautelares y que algunas reglas arbitrales y tratados de inversiones así lo establecen ${ }^{132}$. Cabe citar que el Reglamento de arbitraje de la CNUDMI y del CIADI reconocen la posibilidad del tribunal de establecer cualquier medida para preservar los bienes que puedan satisfacer una adjudicación posterior. En este sentido, el Reglamento de arbitraje de la CNUDMI establece: "Por medida cautelar se entenderá toda medida temporal por la que, en cualquier momento previo a la emisión del laudo por el que se dirima definitivamente la controversia, el tribunal arbitral ordene a una de las partes que, por ejemplo: Proporcione algún medio para preservar bienes que permitan ejecutar todo laudo subsiguiente"133. Mientras que el Reglamento del CIADI reconoce que: "En cualquier etapa una vez incoado el procedimiento, cualquiera de las partes puede solicitar que el Tribunal recomiende la adopción de medidas provisionales para la salvaguardia de sus derechos. La solicitud deberá especificar los derechos que se salvaguardarán, las medidas cuya recomendación se pide, y las circunstancias que hacen necesario el dictado de tales medidas"134, estando en relación con el artículo 47 del Convenio CIADI cuando establece: "Salvo acuerdo en contrario

131 Algunos autores reconocen que, aunque no esté expresamente regulado en las reglas arbitrales, debe considerarse, dentro de las facultades del tribunal arbitral para ordenar medidas provisionales, la inclusión de la caución de las costas; LEW, J.D.M, MISTELIS, L.A., KRÖLL, S., Comparative International Commercial Arbitration, Kluwer Law International, 2003, pp. 600-601; VOSER, N., "New Rules on Domestic Arbitration in Switzerland: Overview of Most Important Changes to the Concordat and Comparison with Chapter 12 PILA", ASA Bulletin, Vol. 28, No. 4, 2010, p. 762 .

132 Véase, Arbitration Rules of the Singapore International Arbitration Centre, Article 24(k), 2013; Arbitration Rules of the Australian Centre for International Commercial Arbitration, Article 33.2(e), 2016; EU-Vietnam Free Trade Agreement, Chapter 8: Trade in Services, Investment and E-Commerce (the EU-Vietnam FTA), Section 3, Article 22.1, 2016; Arbitration Rules of the Singapore International Arbitration Centre, Draft for Public Consultation, Rule 26.k, 2016.

133 Art. 26.2.c del Reglamento de la CNUDMI, 2010.

134 Regla 39.1 del Reglamento del CIADI, 2006. 
de las partes, el Tribunal, si considera que las circunstancias asi lo requieren, podrá recomendar la adopción de aquellas medidas provisionales que considere necesarias para salvaguardar los respectivos derechos de las partes".

Desde el punto de vista comparativo, el estándar para aplicar medidas cautelares establecido en el Reglamento del CIADI es menos exigente que el establecido en el Reglamento de la CNUDMI. Tanto el Convenio como el Reglamento CIADI establecen que las medidas provisionales podrán dictarse por el tribunal si considera que las circunstancias así lo requieren con el objetivo de "salvaguardar los derechos de las partes". Mientras que el Reglamento de la CNUDMI establece que el tribunal, para poder dictar una medida cautelar, debe estar convencido, por un lado, de que existe cierta probabilidad de que se produzca un daño no resarcible adecuadamente por una indemnización por daños y perjuicios si la medida no se ordena, y que tal daño debe ser "significativamente más grave" que el que pudiera sufrir la parte contra la que se dirige la medida una vez otorgada. Y, por otra parte, que exista una posibilidad razonable de que la parte que solicita la caución tenga éxito en los méritos de la demanda, por supuesto, sin un juicio previo por parte del tribunal.

Sin embargo, a pesar de que las exigencias establecidas en las reglas de arbitraje del CIADI carecen de cierta rigurosidad en relación con las establecidas en las reglas de arbitraje de la CNUDMI para dictar una cautio judicatum solvi, los tribunales arbitrales en procedimientos de arbitraje bajo las reglas del CIADI cuando han conocido de una solicitud de caución, han reconocido que dicha solicitud solo puede otorgarse cuando existan circunstancias excepcionales. Requisito imprescindible para ordenar medidas provisionales bajo las Reglas de Arbitraje del CIADI y que le ha valido a los tribunales arbitrales para rechazar todas las solicitudes de caución presentadas hasta la fecha ${ }^{135}$, con la única excepción del caso $R S M$

135 Véase, Alasdair Ross Anderson vs. Republic of Costa Rica, ICSID Case No. ARB(AF)/07/3, paragraph 9, 2010; Plama Consortium Limited vs. República de Bulgaria, Caso CIADI No. ARB/03/24, Orden del Tribunal sobre la Solicitud del Demandante de Medidas Cautelares Urgentes, de fecha 6 de septiembre de 2005; Saipem S.p.A. vs. República Popular de Bangladesh, Caso CIADI No. ARB/05/7, Decisión sobre Jurisdicción y Recomendación de Medidas Cautelares, de fecha 21 de marzo de 2007; Libananco Holdings Co. Limited vs. Turquía, Caso CIADI No. ARB/06/8, Decisión sobre Cuestiones Preliminares, de fecha 23 de junio de 2008; Commerce Group Corp. \& San Sebastian Gold Mines, Inc. vs. El Salvador, Caso CIADI No. ARB/09/17, Decisión sobre la Solicitud de El Salvador de Cautio Judicatum Solvi, de fecha 20 de septiembre de 2012; Burimi S.R.L. and Eagle Games SH. A. vs. República de Albania, Caso CIADI No. ARB/11/18, Orden Procesal No. 2, de fecha 3 de mayo de 2012; Occidental Petroleum Corporation y Occidental Exploration and Production Com- 
Production Corporation vs. Saint Lucia ${ }^{136}$. Por tal motivo, ha resultado muy difícil para los tribunales arbitrales en el arbitraje de inversiones ordenar una caución al reclamante con el objetivo final de garantizar el cobro de los posibles costes adversos surgidos durante el procedimiento por la parte correspondiente.

En otro orden, nos encontramos con la otra parte de la doctrina reacio a admitir como facultades del tribunal el poder decretar al demandante la constitución de una caución, al considerar que el verdadero obligado será finalmente el tercero financiador. Los tribunales arbitrales han recibido una fuerte presión por quienes no defienden tal postura. Esta parte de la doctrina alega, como principal fundamento, que el tercero financiador no es parte del procedimiento, no ha participado en el contrato entre las partes en litigio ${ }^{137} \mathrm{y}$, por tanto, no puede verse obligado bajo los efectos de una orden de caución que emita el tribunal para garantizar los costos adversos generados durante el procedimiento ${ }^{138}$ o una futura condena en costas contra el demandante.

Expuestos hasta aquí los diferentes puntos de vistas existentes en la doctrina en relación con la cuestión, no cabe duda de que la existencia de circunstancias excepcionales se ha convertido en el factor principal para que el tribunal en el ISDS pueda decretar una cautio judicatum solvi.

\section{CONCLUSIONES}

Tanto las ONGs como diversas representaciones de la sociedad civil, han logrado más derechos de participación en los procedimientos

pany vs. República de Ecuador, Caso CIADI No. ARB/06/11, Decisión sobre Medidas Cautelares, de fecha 17 de agosto de 2007; Rachel S Grynberg, Stephen M Grynberg, Miriam Z Grynberg and RSM Production Corporation vs. Government of Grenada, ICSID Case No. ARB/10/6, Decisión sobre la Solicitud del Demandado de Cautio Judicatum Solvi, de fecha 14 de octubre de 2010. Algunos de los casos aquí referenciados por su relevancia serán objeto de análisis en el presente epígrafe.

136 RSM Production Corporation vs. Saint Lucia, ICSID caso n. ${ }^{\circ}$ ARB/12/10, 2012.

137 SCHERER, M., "Third-Party Funding in Arbitration: Out in the Open?", Commercial Dispute Resolution, 2012, p. 57; WAINCYMER, J., Procedure and Evidence in International Arbitration, Kluwer Law International, The Netherlands, 2012, p. 644; SANDROCK, O., "The Cautio Judicatum Solvi in Arbitration Proceedings or The Duty of an Alien Claimant to Provide Security for the Costs of the Defendant", Journal of International Arbitration, Vol. 14, No. 2, 1997, p. 34.

138 DARWAZEH, N., LELEU, A., "Disclosure and Security for Costs or How to Address Imbalances Created by Third-Party Funding", Journal of International Arbitration, Vol. 33 No. 2, 2016, pp. 125-150. 
de arbitraje internacional de inversiones. Especialmente en la aceptación de las comunicaciones amicus curiae, algo que se refleja en las enmiendas del Reglamento de Arbitraje del CIADI, la adopción de las nuevas Reglas de Transparencia de la CNUDMI y la jurisprudencia. Sin embargo, aunque los esfuerzos recientes muestran signos de mejora, el nivel de participación de terceros en el procedimiento sigue siendo limitado.

La participación de terceros no debe verse como un obstáculo al arbitraje internacional en materia de inversión extranjera, sino como una manera de garantizar la transparencia del procedimiento, medida que podría ser complementada con una mayor publicidad de los laudos que en ellos se emitan. Ello permitirá un fortalecimiento y legitimación del ISDS, al complementarse los argumentos de las partes en conflicto en causas de interés público, con los puntos de vista que legítimamente pueden plantear un tercero ajeno a la controversia y que pudiesen verse indirectamente afectados por la solución de la controversia que se trate.

Existe en las reglas de arbitraje institucionales y hasta en los propios TBI - en el caso específico del arbitraje de inversiones-, una ausencia de normas que regulen la práctica de la financiación por terceros en el arbitraje internacional. Esto puede interferir, a la vez, en un equilibrio entre el tercero financiador y el resto de las partes en la relación jurídica, lo que ha propiciado que el tercero financiador se convierta en un eslabón imprescindible para el demandante en el arbitraje internacional, sin restricciones legislativas al respecto.

En la busqueda de una mayor transparencia de los procedimientos de arbitraje con presencia de terceros financiadores, especialmente en el ISDS, se hace necesario una actualización de las reglas arbitrales y que las mismas establezcan: primero, la obligación de revelación del tercero financiador y el acuerdo de financiación a todas las partes involucradas en el caso. Creemos que dicha obligación es muy útil y necesaria, a la luz de las graves consecuencias que un acuerdo de financiación desconocido, no revelado, puede causar al procedimiento arbitral, como puede ser, entre otras, la existencia de posibles conflictos de interés. Y, en segundo lugar, que dicha regulación otorgue mayor competencia al tribunal arbitral para que el mismo pueda pronunciarse respecto al acuerdo de financiación y la relación del tercero financiador con el procedimiento arbitral. De esta manera, se le confiere al tribunal mayor responsabilidad pública y alto nivel de transparencia en el arbitraje internacional. También es necesario que la comunidad 
arbitral internacional asuma un rol más activo en la construcción de un régimen significativo que regule la participación de terceros financiadores en el arbitraje internacional.

La existencia de una regulación a nivel internacional sobre la financiación de terceros no solo favorece la creación de un marco armonizador de las normas procedimentales, sino también de las normas éticas - en relación con el conflicto de interés- en la financiación por terceros. Esto favorecería el establecimiento de estándares mínimos en cuanto a la influencia e interacción del tercero financiador en el procedimiento arbitral, logrando una mayor integridad del procedimiento arbitral con presencia de un tercero financiador.

La financiación por terceros tiene una influencia directa en el procedimiento arbitral, puede ser determinante, en ocasiones, en el aumento de los costes y el prolongado tiempo de duración de los procedimientos arbitrales. Cuestiones que pueden favorecer que el acuerdo de financiación, en un futuro no muy lejano, se convierta en un componente catalizador en la transformación de los litigios internacionales, teniendo efectos significativos en las propias políticas sustantivas y regulatorias de los Estados. Todo ello amerita una mayor coordinación y cooperación internacional en la creación de políticas regulatorias que rijan la financiación por terceros en los procedimientos arbitrales en el ámbito de la litigación internacional.

\section{BIBLIOGRAFÍA}

Adeline, A., Perrin, L., "Third-party funding of arbitration in France", Squire Sanders International Arbitration News, 2013. Disponible en: www.squiresanders.com/files/Publication/bfa63ce49bcd-44fc-8eae-08f219a66927/Presentation/PublicationAttachment/abdbf613-9b29-4060-b2d5-0975f2ab4dbd/International-Arbitration-News-March-2013.PDF.

AFFAKI, G., "A financing is a financing is a financing", en CREMADES, B., Dimolitsa, A. (eds.): Dossier X: Third-party Funding in International Arbitration, ICC Publishing S.A., Paris, 2013.

ANDRÉs ROS, O., SEvila, E., "La financiación por terceros en el arbitraje internacional: regulación, ventajas e inconvenientes", Andersen Tax \& Legal, 2018.

Appelbaum, B., "Lawsuit Loans Add New Risk for the Injured", The New York Times, 2011. Disponible en: www.nytimes. com/2011/01/17/business/17lawsuit.html?pagewanted=all\&_r=0. 
AtTrill, W., "Ethical issues in litigation funding", IMF (Australia), 2009.

Barker, G.R., "Third Party Funding in Australia and Europe", Law and Economics Center, 2011.

BASTIN, L., "Amici Curiae in Investor-State Arbitration: Eight Recent Trends”, Arbitration International, Vol. 30, No. 1, 2014.

- “The Amicus Curiae in Investor-State Arbitration", Camb.J. Int'l \& Comp.L, Vol. 1, 2012.

BAUER Bronstrup, F., "El amicus curiae en la jurisdicción constitucional española", Revista Española de Derecho Constitucional, No. 108, 2016.

Bench Nieuwveld, L., Shannon, V., "Third-Party Funding in International Arbitration”, Kluwer Law International, 2012.

Bert, I.H., "Litigation Finance: What Do Judges Need to Know?", Colum. J.L. \& Soc. Probs, Vol. 45, 2012.

Bertrand, E., "The Brave New World of Arbitration: Third-Party Funding", ASA Bulletin, Vol. 3, 2011.

Bogart, C.P.: "Third Party Funding in Action: Alemanni v Argentina", 2015. Disponible en: http://www.burfordcapital.com.

BOOLIERIS, E., Third-Party Funding: the effect of the growing third-party funding industry in international arbitration on New Zealan, Victoria University of Wellington, 2015.

BorN, G.B, International commercial arbitration, Wolters Kluwer Law \& Business, Primera Edición, The Netherlands, 2009.

Burke Robertson, C., "The Impact of Third-Party Financing on Transnational Litigation", Case Western Reserve Journal of International Law, Vol. 44, 2012.

BRUnNeE, J., Toope, S.J., Legitimacy and Legality in International Law: An Interactional Account, Cambridge University Press, Cambridge, 2010.

Caron, D., Caplan, L.M., The UNCITRAL, Arbitration Rules: A Commentary, Segunda Edición, Oxford, 2013.

Casado Filho, N., "O Financiamento de Arbitragens por terceiros", Direito Internacional em Análise, Vol. 4, 2015.

CHAN, E.: "Funding International Arbitration", New Zealand Law Journal, 2014. 
Christina, K., August, R., “Transparency versus Confidentiality in International Investment Arbitration - The Biwater Gauff Compromise”, Law \& Prac. Int'L cts and Tribunals, Vol. 6, 2007.

Citing Mulheron, R., Cashman, P., "Third Party Funding- A Changing Landscape", Civil Justice Quarterly, No. 3, 2008.

Cremades, B., "Concluding remarks", en Cremades, B., Dimolitsa A. (Eds.): Dossier X: Third-party Funding in International Arbitration, ICC Publishing S.A., Paris, 2013.

- "Third party funding in international arbitration", B. Cremades y Asociados, 2011, Disponible en: https://www.cremades.com/pics/ contenido/File634523783352588756.pdf.

— "Third Party Litigation Funding: Investing in Arbitration", Transnational Dispute Management, Vol. 8, No.1, 2011.

Darwazeh, N., Leleu, A., "Disclosure and Security for Costs or How to Address Imbalances Created by Third-Party Funding", Journal of International Arbitration, Vol. 33 No. 2, 2016.

DE Boulle, T., Third-Party funding in international commercial arbitration, Ghent University, 2014.

De Brabandere, E., "Human Rights Considerations in International Investment Arbitration", en Fitzmaurice, M., Merkouris, P. (Eds), The Interpretation and Application of the European Convention of Human Rights: Legal and Practical Implications, Queen Mary Studies in International Law, London, 2012.

De Brabandere, E., LepeltaK, J., "Third Party Funding in International Investment Arbitration", Grotius Centre for International Legal Studies, No.1, 2012.

De Brabandere, E., "NGOs and the 'Public Interest': The Legality and Rationale of Amicus Curiae Interventions in International Economic and Investment Disputes", Chicago Journal of International Law, Vol. 12, 2011.

De Мот, J., Faure, M.G., Visscher. L., "TPF and Its Alternatives: An Economic Appraisal", Research Conference on Third Party Funding of Litigation, Leiden Law School, 2016.

De Morpurgo, M., "A Comparative Legal and Economic Approach to Third-Party Litigation Funding", Cardozo Journal of International and Comparative Law, Vol. 19, 2011.

Destefano, M., "Nonlawyers Influencing Lawyers: Too Many Cooks in the Kitchen or Stone Soup?", Fordham L. Rev, Vol. 80, 2012. 
Derains Y., "Foreword", en Cremades B.M., Dimolitsa A (Eds.): Dossier X: Third-party Funding in International Arbitration, ICC Publishing S.A., París, 2013.

Dos SANTOS, C., "Third-party funding in international comercial arbitration: a wolf in sheep's clothing?”, ASA Bulletin, Vol. 35, No. 4, 2017.

Eberhardt, P., Olivet, C., "Profiting from injustice. How law firms, arbitrators and financiers are fuelling an investment arbitration boom", Corporate Europe Observatory, 2012.

FACH GÓMEZ, K., "Rethinking the role of amicus curiae in international investment arbitration: how to draw the line favorably for the public interest", Fordham International Law Journal, Vol. 35, 2012.

Febles Pozo, N, "La transparencia y el interés público en el arbitraje de inversiones", Vniversitas, Vol. 69, 2020, p. 4.

FERNÁNDEZ MASIÁ, E., "La financiación por terceros en el arbitraje internacional", Cuadernos de Derecho Transnacional, Vol. 8, No. 2, 2016.

FlaKe, C.R., "Third Party funding in domestic arbitration: champerty or social utility?”, Dispute Resolution Journal, Vol. 70, No. 2, 2012.

FLÉchet C., "Summary report of the first session held", Revue de Droit des Affaires Internationales/International Business Law Journal, No. 2, 2012.

GARBER, S., "Alternative Litigation Financing in the United States", Occasional Paper, 2010. Disponible en: https://www.rand.org/content/dam/rand/pubs/occasional_papers/2010/RAND_OP306.pdf.

FrischKnecht, A., Schmidt, V., "Privilege and Confidentiality in Third Party Funder Due Diligence: The Positions in the United States and Switzerland and the Resulting Expectations Gap in International Arbitration", Transnational Dispute Management, Vol. 8, No.4, 2011.

GLICKMAN, D.R., "Embracing third-party litigation finance", Florida State University Law Review, Vol. 43, 2016.

HonLET, J.C., Recent decisions on third-party funding in investment arbitration", ICSID Review, 2015.

HaRwood, M.K., Batifort, S.N., Trahanas, CH., "Third-Party Funding: security for costs and other key issues", en LEGUM, B. (Ed.), The Investment Treaty Arbitration Review, Law Business Research, Second Edition, 2017.

- "Third-Party Funding: security for costs and other key issues", en LEGUM, B. (Ed.), The Investment Treaty Arbitration Review, Law Business Research, 1st edition, 2016. 
Hodges, CH., Peysner, J., Nurse A., "Litigation Funding: Status and Issues”, Oxford Legal Studies Research Paper, No. 55, 2012.

JAMES, D.F., Odysseus, G.R., "Towards a New World for Investor-State Arbitration through Transparency", N.Y.U. J.Int'l.L, Vol. 48, 2015.

Jansen Calamita, N., "Dispute Settlement Transparency in Europe's Evolving Investment Treaty Policy", J.World Inv. \& Trade, Vol. 15, 2014.

Kantor, M., "Third-Party Funding in International Arbitration: An Essay About New Developments", ICSID Rev., Vol. 24, No. 1, 2009.

KALDERMIS, D., GiBBS, P., "Third-party funding in international arbitration - lessons from litigation?", Kluwer Arbitration, 2014. Disponible en: http://kluwerarbitrationblog.com.

KALICKI, J.E., "Third-Party Funding in Arbitration: Innovations and Limits in Self-Regulation", Kluwer. Arb, 2012, Part 2 of 2. Disponible en: http://kluwerarbitrationblog.com/blog/2012/03/14/thirdparty-funding-in-arbitration-innovations-and-limits-in-self-regulation-part-2-of-2.

KALICKI, J., "Security for Costs in International Arbitration", Transnational Dispute Management, No. 5, 2006. Disponible en: www. transnational-dispute-management.com/article.asp?key=827.

KIDD, J., "To Fund or Not to Fund: The Need for Second-Best Solutions to the Litigation Finance Dilemma", Joumal Law, Economics \& Policy, Vol. 8, 2012.

Kirtley, W., Wietrzykowski, K., "Should an Arbitral Tribunal Order Security for Costs When an Impecunious Claimant Is Relying upon Third-Party Funding?”, J. Int. Arb, Vol. 30, No. 1, 2013.

KHOURI, S., HuRFORD, K., "Third party funding for international arbitration claims: practical tips", Practical Law Company Arbitration, 2014.

Khouri, S., Hurford, K., Bowman, C., "Third party funding in international commercial and treaty arbitration - A panacea or a plague? A discussion of the risks and benefits of third party funding", Transnational Dispute Management, Vol. 8, No.4, 2011.

Lamb, S., Harrison, D., Hew, J., "Recent developments in the law and practice of amicus briefs in investorstate arbitration", Indian Journal of Arbitration Law, Vol. 5, No. 2, 2017.

Lamm, C., HeLlbeck, E.R., "Third-party funding in investor-state arbitration", en Cremades, B.M., Dimolitsa, A. (eds.), Dossier X: Third- 
party Funding in International Arbitration, ICC Publishing S.A., Paris, 2013.

LEGG M., "Litigation Funding in Australia: Identifying and Addressing Conflicts of Interest for Lawyers", Chamber Institute for Legal Reform, 2012.

LEVY, L., Bonnan, R., "Third-party funding: Disclosure, joinder and impact on arbitral proceedings", en CREMADES, B.M., Dimolitsa, A. (eds.): Dossier X: Third-party Funding in International Arbitration, ICC Publishing S.A., Paris, 2013.

Levine, E., "Amicus Curiae in International Investment Arbitration: The Implications of an Increase in Third-Party Participation", Berkeley Journal of International Law, No. 29, 2011.

LEW, J.D.M, Mistelis, L.A., KRöLL, S., Comparative International Commercial Arbitration, Kluwer Law International, 2003.

Lowe R., "Investment arbitration claims could be traded like derivatives”, IBA Global Insigh, 2013. Disponible en: http://www.ibanet. org/Article/Detail.aspx?ArticleUid=02decc8d-bf67-4b86-a023-f2ef2aa4843b.

Lyon, J., "Revolution in Progress: Third-Party Funding of American Litigation”, UCLA Law Review, Vol. 58, 2010

Mann, H., "Reconceptualizing international investment law: Its role in sustainable development", Lewis \& Clark Law Review, Vol. 17, No. 2, 2013.

MANIRUZZAMAN, M., "Third-party funding in international arbitration - A menace or panacea?", Kluwer Arbitration, No. 29, 2012. Disponible en: http://kluwerarbitrationblog.com/blog/2012/12/29/thirdparty-funding-in-international-arbitration-a-menace-or-panacea/.

Marshall, F., ManN, H., "Good Governance and the Rule of Law: Express Rules for investor-state Arbitrations Required", International Institute for Sustainable Development Submissions, 2006.

Mclaughlin, J.H., "Litigation Funding: Charting a Legal and Ethical Course”, Vermont Law Review, Vol. 31, 2007.

Meriam, N., Alrashid, J. W., Laird, J., "Impact of Third Party Funding on Privilege in Litigation and International Arbitration", Dispute Resolution International Law, Vol. 6, 2012.

Miles, C., Zagata Vasani, S., "Case Notes on Third-Party Funding", Global Arbitration Review, Vol. 3, No. 1, 2008. 
Molot, J.T.: "Litigation Finance: A Market Solution to a Procedural Problem”, Georgetown Law Journal, Vol. 99, 2010.

NAVARRo, S., "Cuestiones relativas al third party funding en arbitraje", Arbitraje. Revista de arbitraje comercial y de inversiones, Vol.VII, No. 2, 2014..NG, J.F., "The Role of the Doctrines of Champerty and Maintenance in Arbitration", Arbitration, Vol. 76, 2010

ORTINO, F., "External Transparency of Investment Awards", Inaugural Conference of the Society for International Economic Law (SIEL): New Horizons of International Economic Law, Geneva, 2008. Dispoible en: http://www.ssrn.com/link/SIELInaugural-Conference.html.

PARK, W.W., Rogers, C.R., "Third-Party Funding in International Arbitration: The ICCA Queen-Mary Task Force", 2014. Disponible en: http://ssrn.com/abstract=2507461.

Pascual Vives F.J., "La institución del amicus curiae y el arbitraje de inversiones", Anuario español de derecho internacional privado, Vol. 27, 2011.

Petersmann, E.U., “Justice as Conflict Resolution: Proliferation, Fragmentation, and Decentralization of Dispute Settlement in International Trade", U.PA.J. Int'l. Econ.L., Vol. 27, 2006.

Peterson, L., "Amicus Curiae Interventions: The Tail That Wags the Transparency Dog", Kluwer Arb. Blog, 2010. Disponible en: http:// kluwerarbitrationblog.com/2010/04/27/amicus-curiae-interventions-the-tail-that-wags-thetransparency-dog/.

Petrochilos, G., "Interim Measures under the Revised UNCITRAL Arbitration Rules", ASA Bulletin, Vol. 28, No. 4, 2010.

Polanco Lazo, R., "El capítulo de inversiones en el acuerdo de asociación transpacífico. ¿Una posibilidad de cambio y convergencia?”, 2016. Disponible en: http://ssrn.com/abstract=2515216.

Randazzo, S., "Third Party Funding of Lawsuits Gains Ground But Raises Eyebrows”, Daily J., 2010.

Richmond, D.R., "Other People's Money: The Ethics of Litigation Funding," Mercer Law Review, Vol. 56, No. 2, 2005.

RodaK, M., "It's about Time: A System Thinking Analysis of the Litigation Finance Industry and Its Effect on Settlement", University of Pennsylvania Law Review, 2006.

Rogers, C.A., Ethics in International Arbitration, Oxford University Press, 2014, p. 201; SMITH M., "Mechanics of Third-Party Funding 
Agreements: A Funder's Perspective", en AA.VV.: Third Party Funding in International Arbitration, Kluwer Law International, The Netherlands, 2012.

- "Gamblers, Loan Sharks \& Third-Party Funders", Penn State Law Research Paper, No. 51, 2013. Disponible en: https://ssrn.com/abstract $=2345962$.

- "The Vocation of International Arbitrators", American University International Law Review, Vol. 20, No. 5, 2005

Ross, A., "The Dynamics of Third-Party Funding", Global Arbitration Review, Vol. 7, No. 1, 2012.

Rubins, N., "In God We Trust, All Others Pay Cash: Security for Costs in International Commercial Arbitration", American Review International Arbitration, Vol. 11, 2000.

Ruscalla, G., “Transparency in International Arbitration: Any (Concrete) Need to Codify the Standard?" GroJIL, Vol. 3, No. 1, 2015.

SANDROCK, O., "The Cautio Judicatum Solvi in Arbitration Proceedings or The Duty of an Alien Claimant to Provide Security for the Costs of the Defendant", Journal of International Arbitration, Vol. 14, No. 2, 1997.

Santosuosso, T., Scarlett, R., "Third-Party Funding in Investment Arbitration: Misappropriation of Access to Justice Rhetoric by Global Speculative Finance", Law and Justice in the Americas Working Paper Series, No. 8, 2018. Disponible en: http://lawdigitalcommons.bc.edu/ljawps/8.

Saravanan, A., Subramanian, S.R., "The Participation of Amicus Curiae in Investment Treaty Arbitration", Journal of Civil \& Legal Sciences, Vol. 5, No. 4, 2016.

SeboK, A.J., "Betting on Tort Suits after the Event: From Champerty to Insurance”, DePaul Law Review, Vol. 60, No. 453, 2012.

Sebok A.J, Bradley Wendel W., “Characterizing the Parties' Relationship in Litigation Investment: Contract and Tort Good Faith Norms", Vanderbilt Law Review, Vol. 66, No. 1, 2013.

Scherer, M., Goldsmith, A., Fléchet, C., "Le financement par les tiers des procedures d'arbitrage international —une vue d'Europe Seconde partie: le debat juridique / Third Party Funding of International Arbitration Proceedings- A view from Europe Part II: The Legal Debate", Revue de Droit des Affaires Internationales/International Business Law Journal, 2012. 
SchiLl, S.W., "Crafting the International Economic Order: The Public Function of Investment Treaty Arbitration and Its Significance for the Role of the Arbitrator", Leiden Journal of International Law, Vol. 23, No. 2, 2010.

SCHERER, M., "Third-party funding in international arbitration: Towards mandatory disclosure of funding agreements?", en CREMAdeS, B., Dimolitsa, A. (Eds.): Dossier X: Third-party Funding in International Arbitration, ICC Publishing S.A., Paris, 2013.

- "Third-Party Funding in Arbitration: Out in the Open?", Commercial Dispute Resolution, 2012.

Scherer M., Goldsmith A., "Third Party Funding in International Arbitration in Europe: Part 1-Funders Perspectives", Revue de Droit des Affaires Internationales/International Business Law Journal, No. 2, 2012.

SeIDEL, S., "Fulbrook Primer on Third Party Dispute Finance", Fulbrook Capital Management, LL.C, 2016.

- "Third-party investing in international arbitration claims To invest or not to invest? A daunting question", en CREMADES B.M., DimoLITSA A (Eds.), Third-party Funding in International Arbitration, ICC Publishing S.A., París, 2013.

SEVILA, E., ANDRÉs O., "La financiación por terceros en el arbitraje internacional: regulación, ventajas e inconvenientes", Actualidad Jurídica Aranzadi, No. 941, 2018.

SHANNON, V., "Recent developments in Third-Party Funding", Journal of International Arbitration, 2013.

SHEPHERD, J.M., "Ideal versus reality in third-party litigation financing", Journal Law, Economics \& Policy, Vol. 8, No. 32012.

SHIKAWA T., "Third Party Participation in Investment Treaty Arbitration", International and Comparative Law Quarterly, Vol. 59, No 2, 2010.

SteInitz, M., "Whose claim is it anyway? Third-party Litigation Funding", Transnational Dispute Management, Vol. 8, No. 4, 2011.

Sommer, CH, G., "Los alcances del amicus curiae en el arbitraje internacional de inversiones", Revista de la Facultad Universidad Nacional de Córdoba, Vol. 2, No. 2, 2011.

SteInitz, M., "The Litigation Finance Contract", William \& Mary Law Review, Vol. 54, 2012. 
— "Whose Claim Is It Anyway? Third-Party Litigation Funding", Minnesota Law Review, Vol. 95, 2011.

Subramanian, S.R., "The Participation of Amicus Curiae in Investment Treaty Arbitration", Journal of Civil \& Legal Sciences, Vol. 5, No. 4, 2016.

Trevino, C., "One of Three ICSID Argentine Bond Arbitrations Collapses Due to Lack of Funding", Investment Arbitration Reporter, 2015.

Trusz, J.A., "Full Disclosure? Conflicts of Interest Arising from ThirdParty Funding in International Commercial Arbitration", Georgetown Law Journal, Vol. 101, No. 6, 2013.

VAN Boom W.H., "Third-Party Financing in International Investment Arbitration", OECD, 2011.

- "Financing civil litigation by the European insurance industry", en TUIL, M., VISSCHER, L. (Ed.): New trends in financing civil litigation in Europa: a legal, empirical, and economic analysis, Cheltenham/Northampton: Edward Elgar, 2010.

Van Harten, G., Investment Treaty Arbitration and Public Law, Oxford University Press, Oxford, 2007.

Van Harten, G., Loughlin, M.: "Investment Treaty Arbitration as a Species of Global Administrative Law”, Eur.J.Int'l. L., Vol. 17, No. 1, 2006.

VelJanovski, C., "Third-Party Litigation Funding in Europe”, Journal Law, Economics \& Policy, Vol. 8, No. 3, 2012.

Von Goeler, J., Third-Party Funding in International Arbitration and Its Impact on Procedure, Kluwer Law International, The Netherlands, Vol. 35, 2016.

Voser, N., "New Rules on Domestic Arbitration in Switzerland: Overview of Most Important Changes to the Concordat and Comparison with Chapter 12 PILA", ASA Bulletin, Vol. 28, No. 4, 2010.

Waincymer, J., Procedure and Evidence in International Arbitration, Kluwer Law International, The Netherlands, 2012.

WIRTH, M., "Interim or Preventive Measures in Support of International Arbitraion in Switzerland", ASA Bulletin, Vol. 18, No. 1, 2000.

YU, H.L., "Can third party funding deliver justice in international commercial arbitration?", International Arbitration Law Review, Vol. 20, No. 1, 2017. 